\title{
METAANÁLISIS: EFECTOS DE TÉCNICAS DE PREPARACIÓN MENTAL BASADAS EN IMAGINERÍA - HIPNOSIS SOBRE FLOW Y RENDIMIENTO DEPORTIVO
}

\section{META-ANALYSIS: EFFECTS OF MENTAL PREPARATION TECHNIQUES BASED ON IMAGERY - HYPNOSIS ON FLOW AND ATHLETIC PERFORMANCE} Julián Camilo Garzón Mosquera ${ }^{1}$ y Gerardo Alonso Araya Vargas ${ }^{1,2}$

julian.garzon@ucr.ac.cr; gerardo.araya@ucr.ac.cr

${ }^{1}$ Universidad de Costa Rica

2 Universidad Nacional de Costa Rica

Envío Original: 2019-10-11 Reenviado: 2019-12-17, 2020-02-20 Aceptado: 2020-02-24

Publicado: 2020-03-06

Doi: https://doi.org/10.15517/pensarmov.v18i1.39287

\begin{abstract}
Resumen
El presente estudio tiene como objetivo examinar la efectividad de técnicas psicológicas de preparación mental basadas en imaginería o hipnosis sobre el estado de flow, el rendimiento deportivo y su relación, mediante la técnica meta analítica. Se realizó la revisión de literatura en diez bases de datos durante los meses de agosto a octubre de 2017 , donde se incluyeron 17 estudios; relacionados con actividades deportivas y estudios que presentan mediciones pretestpostest. Se analizó un TE con diferencia de medias bajo modelo aleatorio. Obteniendo un total de 53 tamaños de efecto (TE) para estado de flow y 62 tamaños de efecto (TE) para rendimiento. Se encontró un TE positivo para el estado de flow (TE= .782; $\mathrm{n}=52 ; p<.001)$ y para el rendimiento $(\mathrm{TE}=.339 ; \mathrm{n}=60 ; p<.05)$, se comprueba la relación positiva entre las dos variables $(\rho=.312 ; p<.05)$; se establece que variables más relevantes que moderan el tamaño de efecto del estado de flow y el rendimiento son: edad, años de experiencia deportiva, sexo.
\end{abstract}


Se concluye que la imaginería es la técnica psicológica más efectiva para incrementar el estado de flow y el rendimiento, se recomienda realizar investigaciones con diseño experimental puro y contemplar la medición multi-dimensional del estado de flow.

Palabras claves: estado de flow, rendimiento, imaginería, hipnosis, metaanálisis

\begin{abstract}
The present study aims to examine the effectiveness of psychological mental preparation techniques based on imagery or hypnosis on the state of flow, sports performance, and their relationship, through the meta-analytic technique. The literature review was conducted in ten databases during the months of August to October 2017, in which 17 studies were included; related to sports activities and studies that present pretest-posttest measurements. TE was analyzed with a difference of means under a random model. A total of 53 effect sizes (TE) for flow status and 62 effect sizes (TE) for performance were obtained. A positive TE was found for the flow state $(T E=.782, n=52, p<.001)$ and for the performance $(T E=.339 ; n=60 ; p<.05)$, the positive relationship was verified between the two variables $(\rho=.312, p<.05)$, It is established that the most relevant variables that moderate the effect size of the flow state and performance are: age, years of sports experience and sex. It's concluded that the imagery is the most effective psychological technique to increase the state of flow and performance; it's recommended to carry out research with pure experimental design and contemplate the multidimensional measurement of the state of flow.
\end{abstract}

Key words: flow state, performance, imagery, hypnosis, meta-analysis 


\section{Introducción}

Esta relación interna y externa de estado de flow - rendimiento, es el equilibrio deseado en la satisfacción plena como deportista. Csikszentmihalyi (1975) afirma que son muchas las características cognitivas que encierran al estado de flow; pero es correcto afirmar la existencia de nueve dimensiones : ( 1 ) claridad de meta, ( 2 ) Feedback inmediato de lo óptimo y eficaz que se está realizando la acción, ( 3 ) una atención focalizada en la tarea, ( 4 ) un balance entre las oportunidades de acción ( desafío ) y la capacidad de actuar ( habilidad ), ( 5 ) exclusión de los contenidos irrelevantes de la conciencia, ( 6 ) Fusión acción - atención o automatismo, ( 7 ) un sentido de control sobre la actividad, ( 8 ) una distorsión en el sentido del tiempo ( usualmente las horas pasan como si fueran minutos ) y ( 9 ) un sentimiento de que la actividad es intrínsecamente gratificante (experiencia autotélica).

Experimentar las diferentes dimensiones en conjunto durante determinado espacio de tiempo, canalizan al sujeto en la relación de un equilibrio desafío - habilidad; Nakamura y Csikszentmihalyi (2002), destacan la dimensión desafío - habilidad para ingresar en la zona o canal de flow, se contempla un plano entre las puntuaciones para desafío y habilidad donde a mayor puntuación en ambas dimensiones, se logra tener una mayor probabilidad de ingresar al canal de flow.

Existen técnicas psicológicas propicias para afectar el estado de flow, entre las que se destaca la hipnosis, siendo una modalidad de la conciencia en vigilia donde se predomina la focalización de la atención (Cabrera Macías et al., 2013), asimismo, Montgomery y Schnur (2005) definen la hipnosis bajo el criterio de la American Psychological Association (APA) como:

Un estado mental o un grupo de actitudes generadas a través de un procedimiento llamado inducción hipnótica, es un conjunto de procedimientos que generan un contexto 
donde se facilita el cumplimiento de las sugestiones en ciertas personas, usualmente se compone de una serie de instrucciones y sugerencias preliminares (p.8).

A su vez, la imaginería es considerada un proceso y técnica psicológica para mejorar rendimiento. Como lo explican Vealey y Greenleaf (2010), la imaginería es una técnica psicológica intrínseca de la persona, pero con un proceso externo (rendimiento), haciendo uso en totalidad de los sentidos logrando recrear experiencias en la mente.

El estado de flow es un tema de investigación en el rendimiento deportivo que proliferó en la década de los 90 (Jackson, 1992, 1995; Jackson, Ford, Kimiecik y Marsh, 1998; Jackson y Marsh, 1996; Jackson y Roberts, 1992); en estos primeros estudios se demostró mejora en el rendimiento cuando los atletas presentaban estados de flow durante su práctica deportiva. Un factor clave para esta línea de investigación, fue el desarrollo de instrumentos de medición del estado de flow para ser aplicadas tanto en el entrenamiento como en la competición (Jackson y Marsh, 1996; Jackson y Eklund, 2004; Jackson, Martin y Eklund, 2008). Entre los hallazgos científicos, se ha demostrado que el flow presenta una conjugación de dimensiones que pueden llegar a explicar la naturaleza de este constructo psicológico. Koehn, Morris y Watt (2014a) describieron una relación positiva y significativa tanto en el estado de flow, aumentando los puntos obtenidos en la prueba (138.43 a 161.33), como en el rendimiento deportivo en una prueba motora donde se aumentan los valores de pre a post (7.85 a 13.03); en tenistas con un período de 6 semanas de intervención en imaginería. Asimismo, Técnicas psicológicas como la hipnosis, han sido utilizada por autores como Pates, Cummings y Maynard (2002) quienes evidencian en una muestra de basquetbolistas universitarios, un aumento significativo tanto en el estado de flow $(130-144)$ como en el rendimiento de tiro de tres puntos $(30-36)$, tras realizar una intervención de hipnosis.

La efectividad de las técnicas psicológicas que pueden inducir, predecir o aumentar los estados de flow y el rendimiento deportivo, presentan valores en algunos casos de efectividad frente al 
rendimiento; sin embargo, aún se presentan dudas para su generalización a nivel deportivo. Por ende, es oportuno la realización de un metaanálisis, el cual pueda brindar mayor certeza académica, consenso y futuros estudios sobre el tipo de intervenciones psicológicas que pueden afectar positivamente el estado de flow y a la vez beneficiar al rendimiento deportivo, examinando además la participación de algunas variables moderadoras de estos efectos. Como objetivo principal se establece examinar la efectividad de estas técnicas psicológicas para afectar el estado de flow y el rendimiento deportivo, seguidamente identificar que variables moderadoras están involucradas y finalmente comprobar la relación que existe entre el estado de flow y el rendimiento deportivo en estudios experimentales que involucran técnicas psicológicas de componentes cognitivos o afectivos como imaginería o hipnosis.

\section{Metodología}

Siguiendo la declaración PRISMA, para el desarrollo metodológico de metaanálisis (2010) se procede con:

\section{Búsqueda de literatura}

Se estableció una frase booleana, con las diferentes palabras claves que comprenden los conceptos relacionados con el tema de investigación (Flow state OR optimal experience) AND (performance) AND (sport) AND (imagery OR hypnosis) NOT blood. La búsqueda se realizó tanto en inglés como en español.

Las bases de datos que se utilizaron, se relacionaron al campo deportivo y psicológico y su combinación, las mismas fueron: EBSCOhost (fuente académica, PSYCARTICLES, MEDLINE Full text, SPORTDiscus Full text), Feminism \& Psychology, JSTOR AAF, PsycINFO, ScienceDirect: Psychology, Springer Link. 
Se presentan los siguientes criterios de elegibilidad. El artículo debía estar publicado, presentar un diseño donde se midiera el estado de flow y el rendimiento deportivo, haber realizado una intervención con estrategias psicológicas (imaginería o hipnosis), presentar promedios y desviaciones estándar. No tener restricción de edad, sexo o modalidad deportiva; de igual manera, no tener restricciones con el año de publicación, como también, estar en idioma inglés o español.

Los estudios que no presentaron uno o más de estos criterios se excluyeron para la elaboración de este metaanálisis como se presenta en el flujograma de la figura 1.

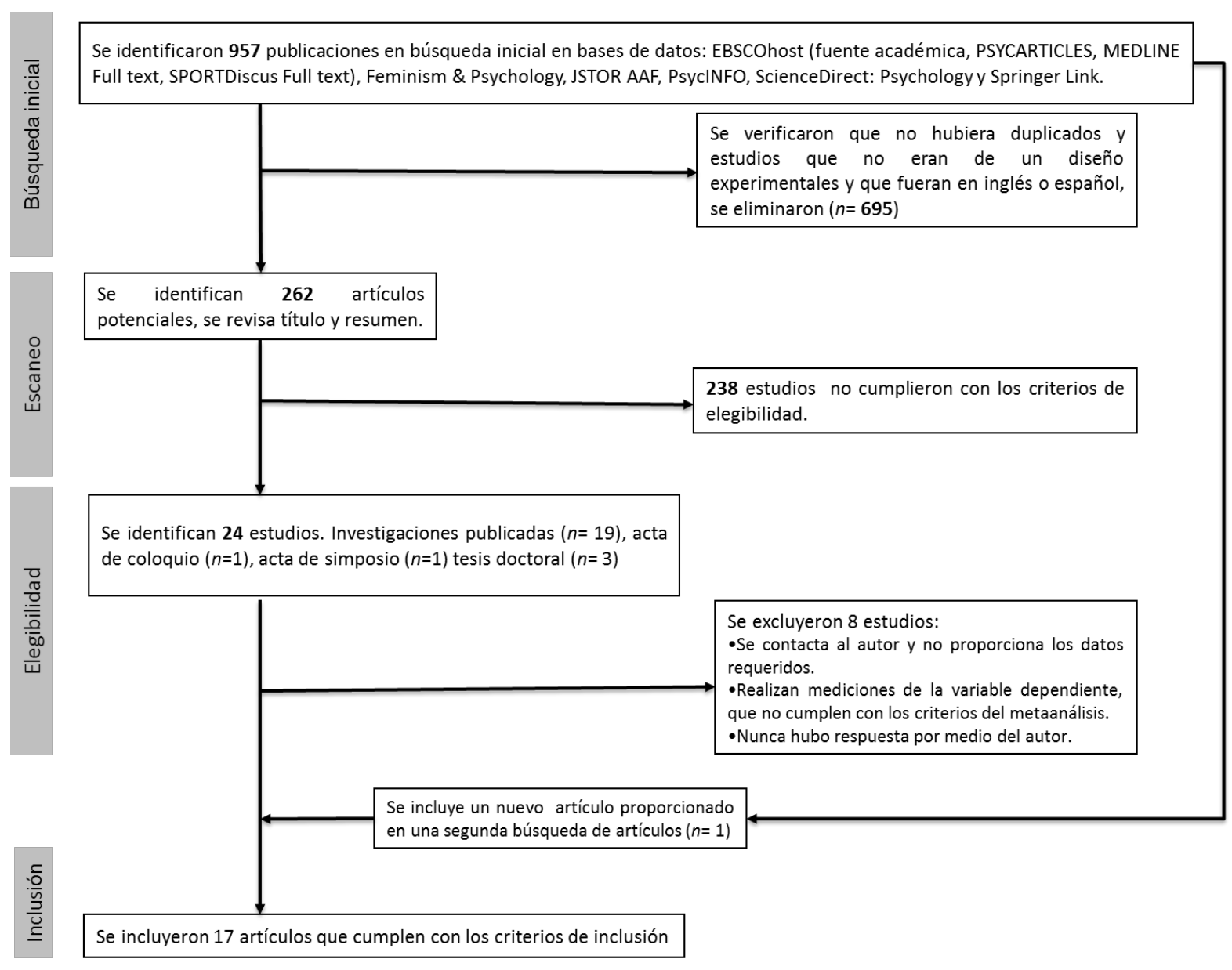

Figura 1. Flujograma. Fuente: elaboración propia. 


\section{Variables moderadoras}

Variables moderadoras, que se tomaron en cuenta al realizar esta investigación son: años de experiencia deportiva de los sujetos, edad, sexo, modalidad deportiva (individual o de conjunto), edad, medición de flow y medición de rendimiento. Estudios que respaldan escoger dichas variables moderadoras se pueden contemplar en Koehn y Díaz-Ocejo (2016) y Pain, Harwood y Anderson (2011).

Procedimiento para el cálculo del tamaño de efecto individual para el diseño intragrupo

Se llevó a cabo el cálculo de los diferentes tamaños de efecto individuales (TE) mediante la fórmula de delta propuesta por Thomas y French (1986). Se ilustra en la figura 2 TE (1), varianza (2). Seguido a esto, se realiza la corrección del TE y la varianza con un factor de corrección c (3) de Borenstein, Hedges, Higgins y Rothstein (2011). De igual manera, se calcularon los intervalos de confianza al 95\% ( $p<.05)(4)$. Al finalizar este proceso, se realizó una verificación del signo corregido del TE para que tenga una coherencia con relación a los resultados que plantea cada grupo.

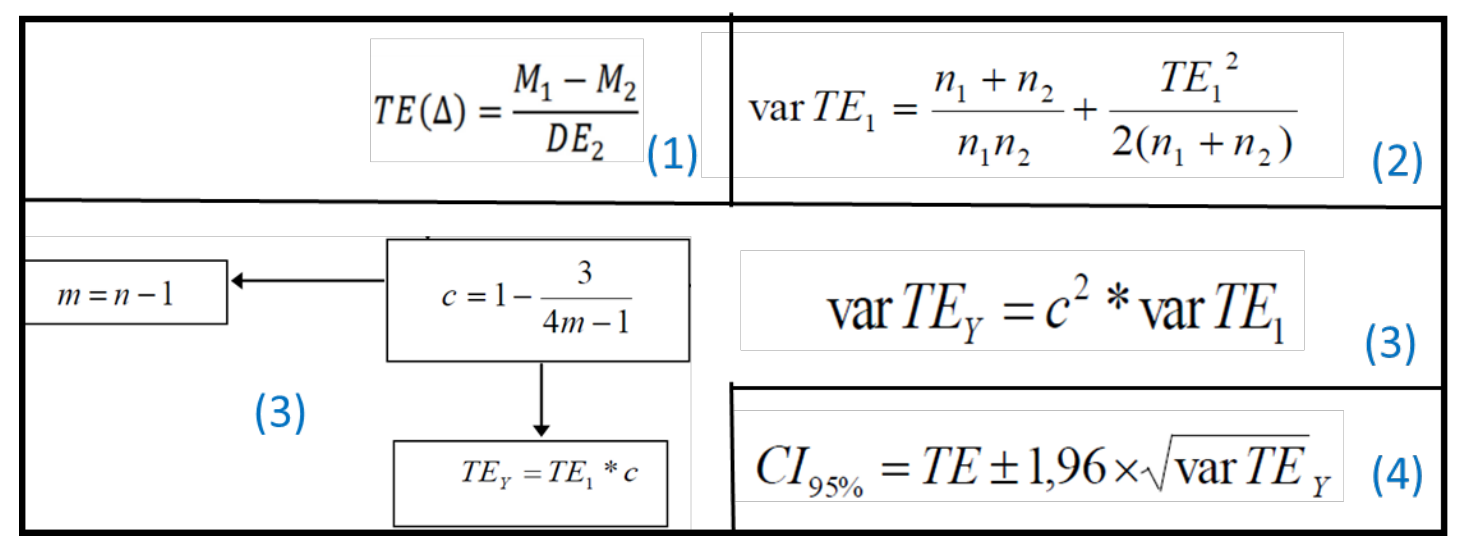

Figura 2. Formulas tamaño de efecto individual para el diseño intra-grupo. Fuente: elaboración propia. 
Cálculo tamaño de efecto global ponderado para modelo de efectos aleatorios

El tamaño de efecto global ponderado para el modelo de efectos aleatorios propuesto por Borenstein et al. (2011), establece una secuencia de pasos descritos en la figura 3. Primeramente, se calcula el inverso de la varianza (w) para cada estudio (1); seguido a este paso, se establece (Q) como prueba de heterogeneidad (2) y T2 (3), así se logra llegar a tener la fórmula de $\left(\mathrm{W}^{*}\right)$ para la varianza del tamaño de efecto y T2 (4); se realiza la sumatoria de $\left(\mathrm{W}^{*}\right.$ $x$ TE) y la sumatoria de $\left(W^{*}\right)$. Resultando en el tamaño de efecto global ponderado del modelo de efectos aleatorios. Finalmente, se realiza la división (5), para establecer la varianza de $\mathrm{W}^{*}$ (6) desarrollando el análisis de intervalos de confianza al 95\% tanto negativo como positivo (7).

Posteriormente se analiza Z, para determinar si él TE es significativo o no descrito en la figura $\underline{4}$.

\begin{tabular}{|c|c|c|}
\hline$w=\frac{1}{\operatorname{var} T E_{Y}}$ & \multicolumn{2}{|c|}{$Q=\sum\left(w \times T E_{Y}^{2}\right)-\frac{\left[\sum\left(w \times T E_{Y}\right)\right]^{2}}{\sum w}$} \\
\hline$T^{2}=\frac{Q-(k-1)}{\sum w-\left(\frac{\sum w^{2}}{\sum w}\right)}$ & $w^{*}=\frac{1}{\operatorname{var} T E_{Y}+T^{2}}$ & $\overline{T E}=\frac{\sum\left(w^{*} \times T E\right)}{\sum w^{*}}$ \\
\hline $\operatorname{var} \overline{T E}=\frac{1}{\sum w^{*}}$ & $I C_{95 \%}=T E$ & $6 \times \sqrt{\operatorname{var} \overline{T E}}$ \\
\hline
\end{tabular}

Figura 3. Formulas tamaño de efecto global ponderado, modelo de efectos aleatorio. Fuente: elaboración propia.

$$
z=\frac{T E}{\sqrt{\text { varTE }}}
$$

Figura 4. Formula Z. Fuente: elaboración propia. 
Prueba de $I^{2}$

Se realiza el análisis del índice $\mathrm{I}^{2}$ de la figura 5 , el cual es un dato estadístico que representa en que porcentaje los TE individuales son heterogéneos (Borenstein et al., 2011).

$$
I^{2}=\frac{Q-g l}{Q} * 100
$$

Figura 5. Formula de heterogeneidad $\mathrm{I}^{2}$. Fuente: elaboración propia.

Prueba de sesgo

Se aplicara estudios de trabajos archivados $K_{0}$ de la figura 6 definido por Orwin (1983), necesaria para conocer la cantidad de TE no significativos o pequeños. Los cuales son necesarios para reducir él TE global significativo a un TE nulo $\left(d_{2}=0.20\right)$.

$$
K_{o}=\frac{K\left(\mathrm{~d}_{1}-\mathrm{d}_{2}\right)}{d_{2}}
$$

Figura 6. Formula de efectos de trabajos archivados $\mathrm{K}_{0}$. Fuente: elaboración propia.

Análisis de datos

Se pruebas correlacionales (Spearman), para examinar la relación entre los tamaños de efecto y las variables moderadoras métricas, también pruebas comparativas para examinar el posible efecto de variables moderadoras no métricas con análisis de varianza (ANOVA) de una vía; cuando se halló diferencia significativa, se aplicó la prueba de Post Hoc de Tukey. Para dichos cálculos se utilizó el programa estadístico IBM-SPSS versión $24 \AA$, el programa de hoja de 
cálculo de Microsoft Excel ${ }^{\circledR}$ y Rstudio para la elaboración de forestplot y gráfico de embudo respectivos.

\section{Resultados}

Se establecieron en la búsqueda final, 17 estudios de los cuales se codificaron 53 tamaños de efecto para estado de flow y 62 tamaños de efecto para rendimiento, para una muestra total de 189 sujetos. Las características de los estudios se describen en la tabla 1.

\section{Tabla 1.}

Características descriptivas de estudios incluidos en el metaanálisis.

\begin{tabular}{|c|c|c|c|c|c|c|c|}
\hline $\begin{array}{l}\text { Autor } \\
\text { (año) }\end{array}$ & país & $\begin{array}{l}\text { Sujetos } \\
(\mathrm{n})\end{array}$ & edad & Deporte & $\begin{array}{l}\text { Medición } \\
\text { flow }\end{array}$ & $\begin{array}{c}\text { Medición } \\
\text { rendimiento }\end{array}$ & Intervención \\
\hline $\begin{array}{l}\text { Pates et } \\
\text { al. }(\underline{2003})\end{array}$ & GBR & 3 & 20 & Netball & $\begin{array}{c}\text { (Jackson y } \\
\text { Marsh, 1996) }\end{array}$ & R.PM & IMA \\
\hline $\begin{array}{c}\text { Lindsay et } \\
\text { al. } \\
(\underline{2005})\end{array}$ & GBR & 2 & 25.3 & $\begin{array}{l}\text { Ciclismo } \\
\text { ruta }\end{array}$ & $\begin{array}{c}\text { (Jackson y } \\
\text { Marsh, 1996) }\end{array}$ & $\mathrm{RC}$ & HIP \\
\hline $\begin{array}{l}\text { Pates et } \\
\text { al. }(\underline{2000})\end{array}$ & GBR & 3 & 21 & Golf & $\begin{array}{c}\text { (Jackson y } \\
\text { Marsh, 1996) }\end{array}$ & R.PM & HIP \\
\hline 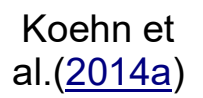 & AUS & 4 & 14 & tenis & $\begin{array}{c}\text { (Jackson y } \\
\text { Eklund, 2002) }\end{array}$ & R.PD & IMA \\
\hline $\begin{array}{l}\text { Pain et al. } \\
(\underline{2011)})\end{array}$ & GBR & 5 & 20.9 & Futbol & $\begin{array}{c}\text { (Jackson y } \\
\text { Marsh, 1996) }\end{array}$ & R.SD & IMA \\
\hline $\begin{array}{l}\text { Pates et } \\
\text { al. (2012) }\end{array}$ & GBR & 3 & 33.5 & Golf & $\begin{array}{c}\text { (Jackson y } \\
\text { Eklund, 2002) }\end{array}$ & R.PD & CC \\
\hline $\begin{array}{c}\text { Pates et } \\
\text { al. } \\
(\underline{2013})\end{array}$ & GBR & 1 & 22 & Golf & $\begin{array}{l}\text { (Jackson y } \\
\text { Eklund, 2002) }\end{array}$ & R.PD & HIP \\
\hline $\begin{array}{l}\text { Pates } \\
(\underline{2013})\end{array}$ & GBR & 1 & 52 & Golf & $\begin{array}{c}\text { (Jackson y } \\
\text { Eklund, 2002) }\end{array}$ & R.PD & HIP \\
\hline $\begin{array}{c}\text { Pates et } \\
\text { al. } \\
(\underline{2002)})\end{array}$ & GBR & 5 & 20.2 & $\begin{array}{c}\text { Basketba } \\
\text { II }\end{array}$ & $\begin{array}{c}\text { (Jackson y } \\
\text { Marsh, 1996) }\end{array}$ & R.PM & HIP \\
\hline
\end{tabular}




\begin{tabular}{|c|c|c|c|c|c|c|c|}
\hline $\begin{array}{l}\text { Nicholls et } \\
\text { al. }(\underline{2005})\end{array}$ & GBR & 4 & 22 & Golf & $\begin{array}{l}\text { (Jackson y } \\
\text { Eklund, 2002) }\end{array}$ & R.PM & IMA \\
\hline $\begin{array}{l}\text { Welo } \\
(\underline{2009)})\end{array}$ & USA & 30 & 20 & $\begin{array}{c}\text { Basketba } \\
\|\end{array}$ & $\begin{array}{c}\text { (Jackson y } \\
\text { Marsh, 1996) }\end{array}$ & R.PM & IMA \\
\hline $\begin{array}{l}\text { Sardon et } \\
\text { al. }(\underline{2016})\end{array}$ & MAS & 8 & 20 & Tenis & $\begin{array}{c}\text { (Jackson y } \\
\text { Eklund, 2002) }\end{array}$ & R.PM & IMA \\
\hline $\begin{array}{l}\text { Jeong } \\
(\underline{2012})\end{array}$ & KOR & 64 & 20 & $\begin{array}{c}\text { Bailarín } \\
\text { danza }\end{array}$ & $\begin{array}{c}\text { (FSS-2; } \\
\text { Jackson y } \\
\text { Eklund, 2004) }\end{array}$ & $\begin{array}{l}\text { R.SE } \\
\text { R.SD }\end{array}$ & IMA \\
\hline $\begin{array}{l}\text { Stefan } \\
\text { Koehn } \\
(\underline{2014 b)}\end{array}$ & AUS & 4 & 14.5 & Tenis & $\begin{array}{c}\text { (Jackson y } \\
\text { Marsh, 1996) }\end{array}$ & R.PD & IMA \\
\hline $\begin{array}{l}\text { Pates et } \\
\text { al. (2001) }\end{array}$ & GBR & 5 & 21 & Golf & $\begin{array}{c}\text { (Jackson y } \\
\text { Marsh, 1996) }\end{array}$ & R.PM & HIP \\
\hline $\begin{array}{c}\text { Vasquez } \\
(\underline{\underline{2006}})\end{array}$ & USA & 31 & 23.3 & $\begin{array}{c}\text { Basketba } \\
\text { II }\end{array}$ & $\begin{array}{c}\text { (Jackson y } \\
\text { Eklund, 2002) }\end{array}$ & R.PM & HIP \\
\hline $\begin{array}{l}\text { Pates et } \\
\text { al. (2002) }\end{array}$ & GBR & 4 & 21.2 & $\begin{array}{c}\text { bádminto } \\
n\end{array}$ & $\begin{array}{c}\text { (Jackson y } \\
\text { Marsh, 1996) }\end{array}$ & R.PD & HIP \\
\hline
\end{tabular}

Notas: Reino unido: GBR, Australia: AUS, Estados unidos: USA, Malasia: MAS, corea del sur: KOR; R.PD: Rendimiento pruebas deportivas, R.PM: Rendimiento pruebas motoras, R.SE: Rendimiento subjetivo entrenador, R.SD: Rendimiento subjetivo del deportista, RC: Resultado en competencia; HIP: Hipnosis, IMA: Imaginería. Fuente: elaboración propia.

\section{Tamaño de efecto global}

Estado de flow: el estado de flow, tanto para grupo experimental con $(n=52)$ y el grupo control $(n=1)$; con 17 estudios incluidos, no se logran establecer las diferencias reales entre los grupos dado que, solo se cuenta con un TE de grupo control frente a 52 de los grupos experimentales. Se obtiene Para el grupo experimental un tamaño de efecto $(\mathrm{TE}=.782 ; p<.001)$ y para el único grupo control $(\mathrm{TE}=.093 ; p=.515)$. Se interpreta que, las intervenciones psicológicas de técnicas de preparación mental basadas en imaginería o hipnosis, tienen un impacto positivo y 
significativo. Sin embargo, no se pudo determinar las diferencias entre grupos limitando los resultados a la interpretación individual del grupo experimental en su TE.

Rendimiento: el rendimiento, tanto para grupo experimental $(n=60)$ y grupo control $(n=2)$, de los 17 estudios incluidos se evidencia que sí existen diferencias significativas $(p<.05)$. Para grupo experimental $(\mathrm{TE}=.789 ; p<.05)$ y para grupo control $(\mathrm{TE}=-.034 ; p>.05)$. Se concluye que las intervenciones psicológicas de técnicas de preparación mental basadas en imaginería o hipnosis, tiene un impacto positivo y significativo sobre la mejora en el rendimiento en actividades deportivas como se observa en la figura 7.

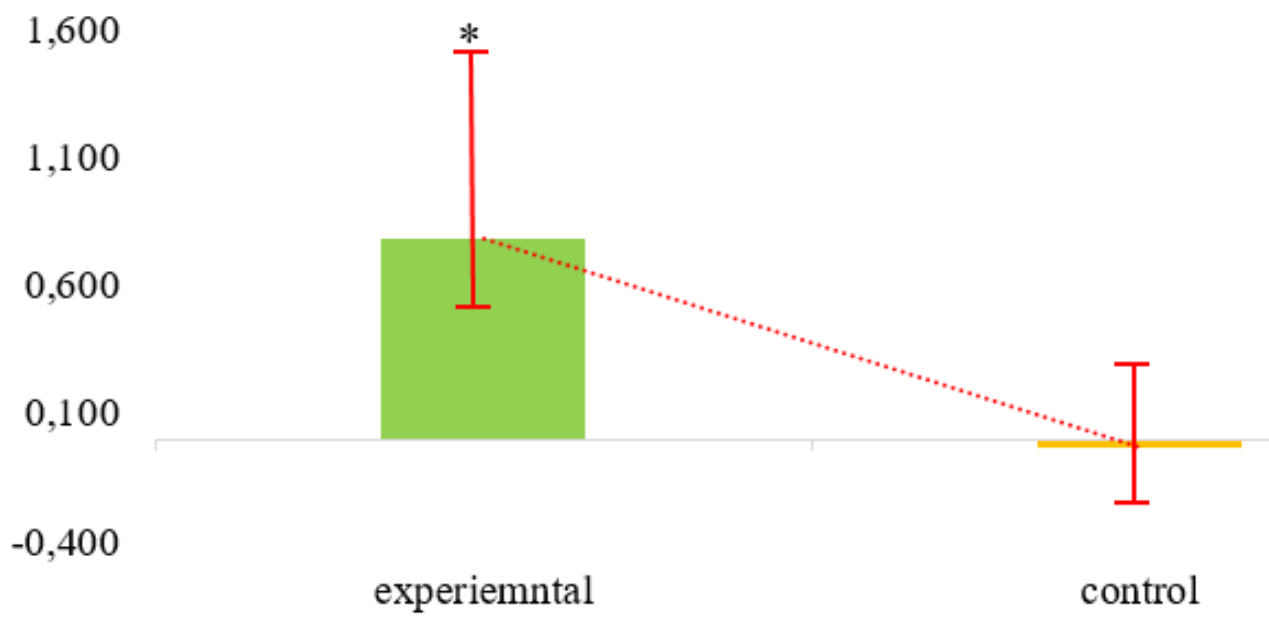

Figura 7. Tamaño de efecto global para grupo experimental TE=.789 $\left({ }^{*} p<.05\right)$. Fuente: elaboración propia.

La prueba de trabajos archivados para la variable estado de flow con $\mathrm{TE}=.782$, para convertirlo a un tamaño de efecto nulo se requiere de 151 tamaños de efecto no significativos. Para la variable rendimiento $\mathrm{TE}=.339$, se requiere de 41 tamaños de efecto no significativos.

El gráfico de embudo presenta una distribución asimétrica para estado de flow, lo que demuestra en la figura 8, que no incluyen todos los estudios relevantes. La regresión de Egger 
arroja que se presenta una distribución simétrica $(\mathrm{t}=1.791 ; \mathrm{gl}=51 ; p=.07)$ y no hay sesgo en general.

El gráfico de embudo presenta una distribución asimétrica para rendimiento, lo que demuestra que el estudio no incluye todos los estudios relevantes en el diseño intragrupo de la figura 9. La regresión de Egger confirma que el gráfico presenta distribución asimétrica $(t=3.788 ; g l=60$; $p<.001)$ y que existe sesgo general. Posiblemente sea ocasionado, por la forma en que se está reportando en la literatura con relación a la variable rendimiento en este tipo de estudios de intervenciones psicológicas, donde conjuntamente son observadas las variables de estado de flow y rendimiento. Asimismo, las figuras $(\underline{10}, \underline{11}$ y $\underline{12})$ presentan el forestplot resultante.

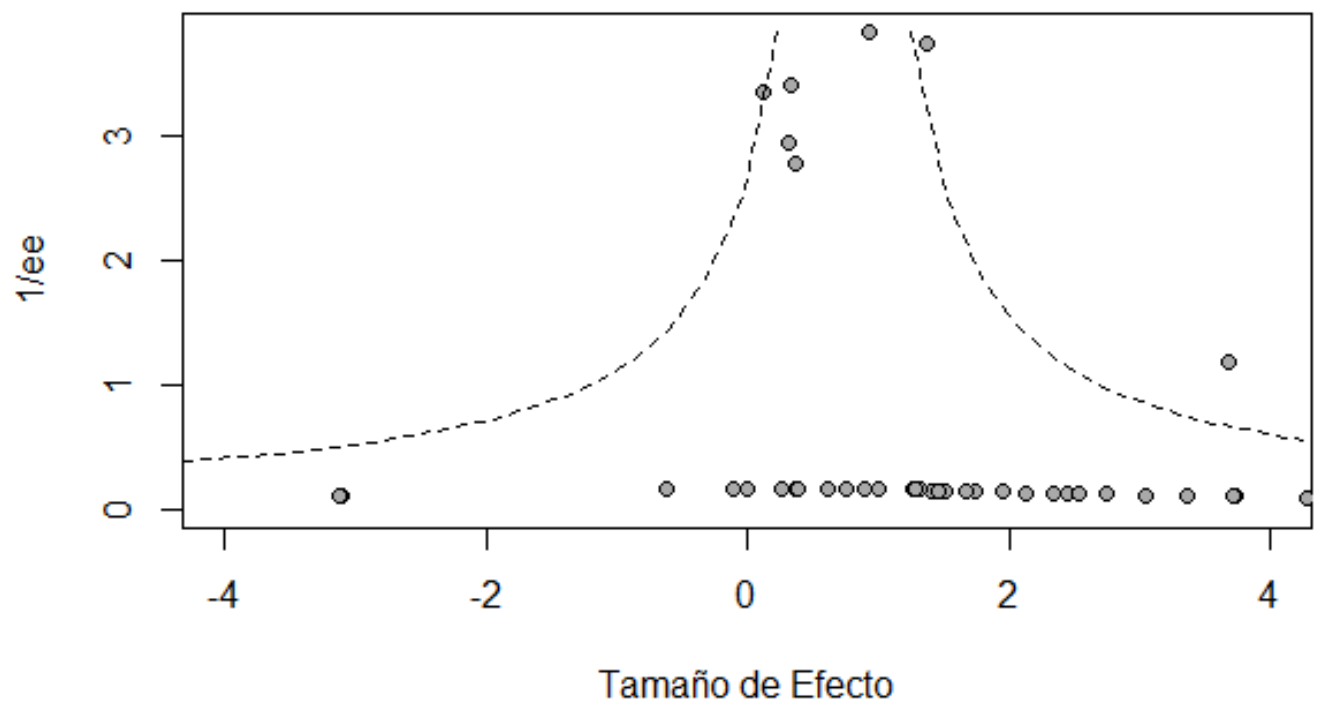

Figura 8. Gráfico de embudo para el diseño intragrupo, variable estado de Flow. La regresión de Egger, demuestra que no existe sesgo $(p=.07)$. Elaboración propia mediante el programa estadístico Rstudio. 


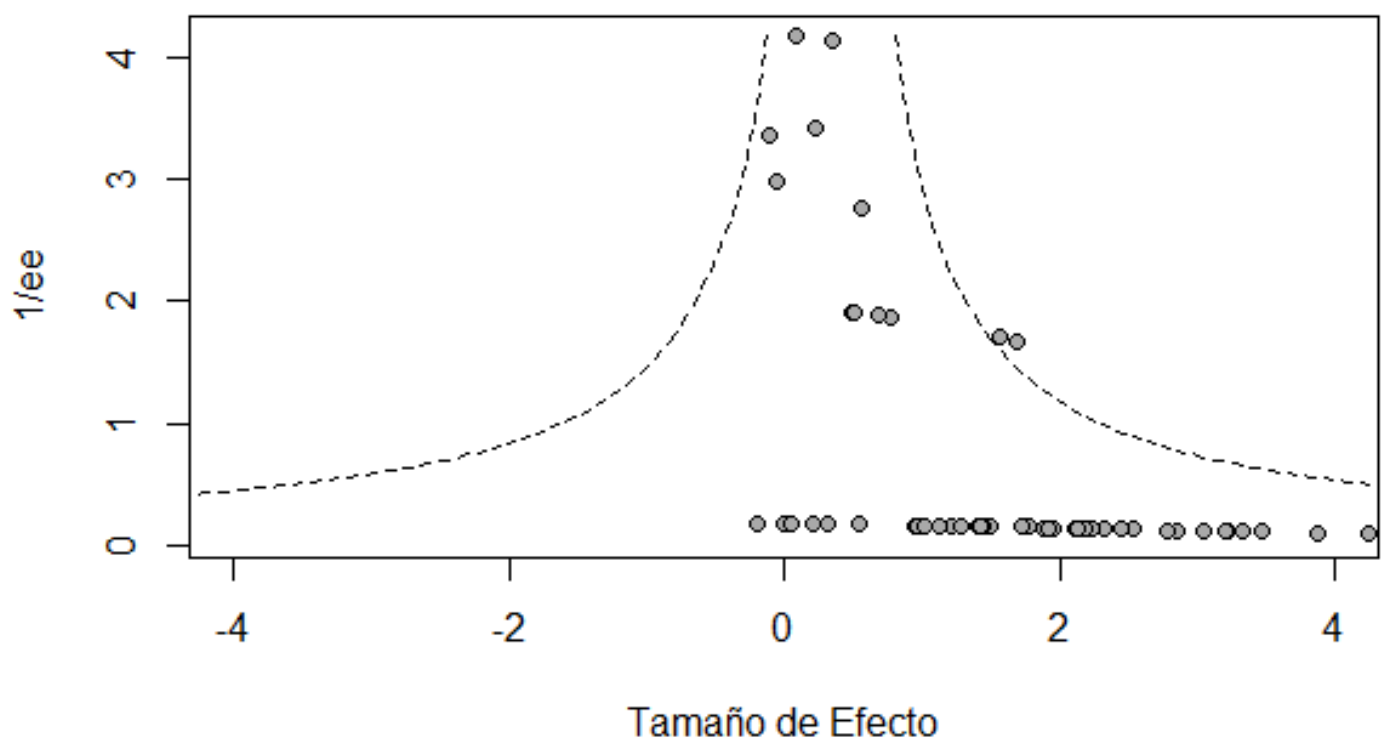

Figura 9. Gráfico de embudo para el diseño intragrupo, variable estado de rendimiento. La regresión de Egger, demuestra que existe sesgo $(p<.001)$. Elaboración propia mediante el programa estadístico Rstudio. 


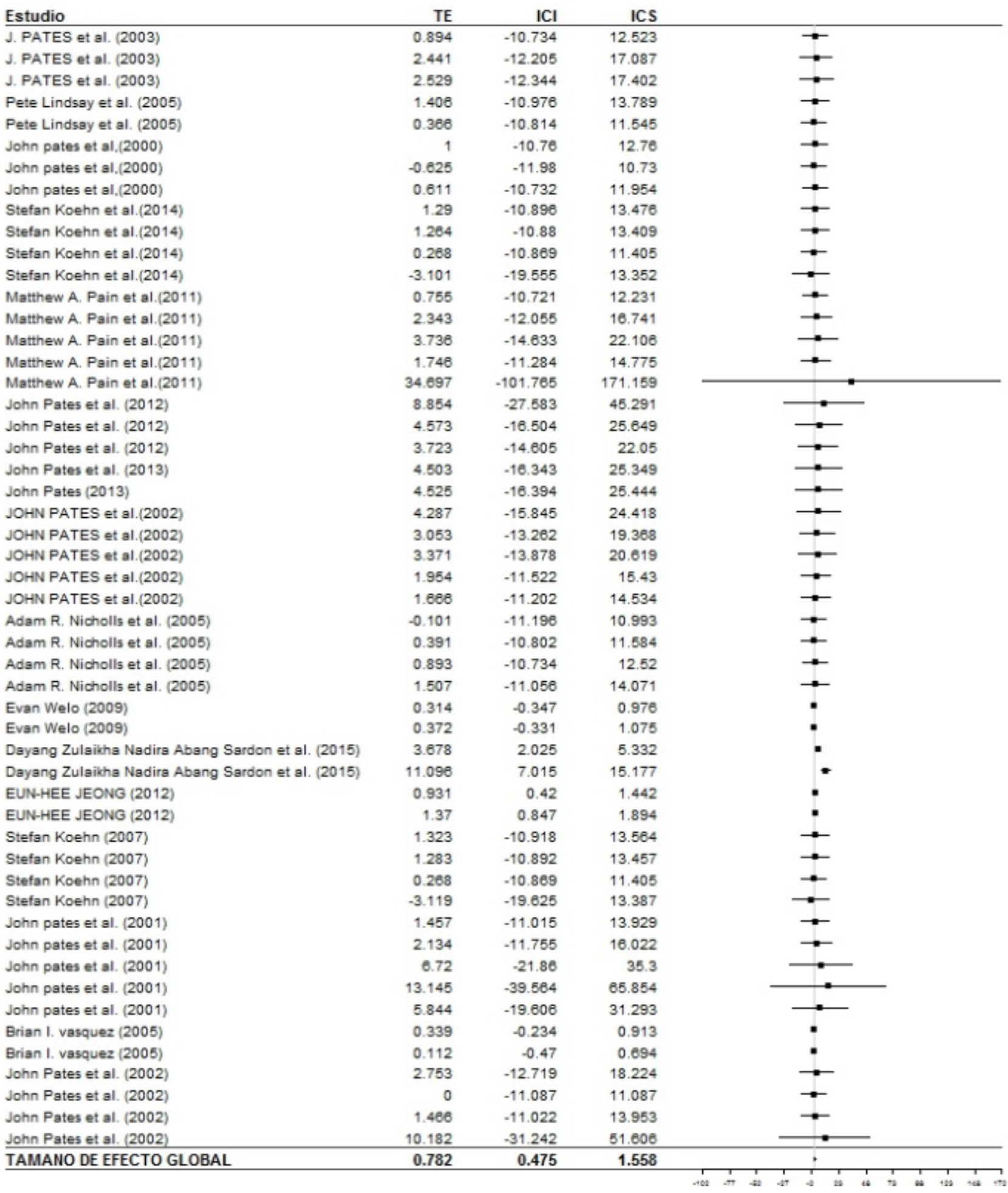

Figura 10. Forest plot de grupo experimental, para la variable Flow; heterogeneidad $(\mathrm{Q})=$ 54.948, sesgo de publicación $\left(K_{0}\right)=151$. Intervalo de confianza al 95\%. Siglas: TE = tamaño de efecto; $\mathrm{ICI}=$ intervalo de confianza inferior; ICS = intervalo de confianza superior. Elaboración propia mediante el programa estadístico Rstudio. 


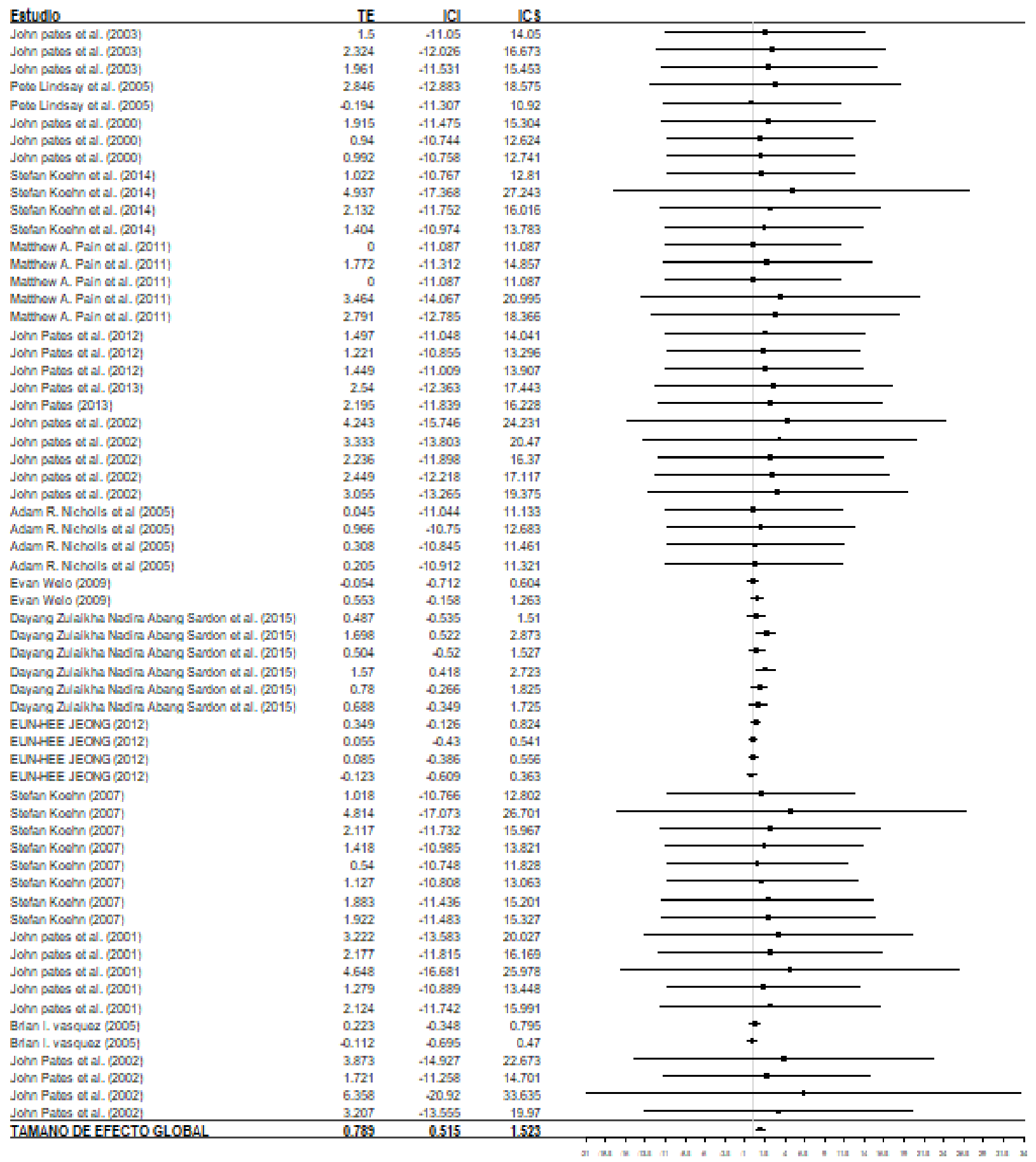

Figura 11. Forest plot de grupo experimental, para la variable rendimiento; heterogeneidad $(\mathrm{Q})=$ 19.053, sesgo de publicación $\left(K_{0}\right)=41$. Intervalo de confianza al 95\%. Siglas: TE = tamaño de efecto; $\mathrm{ICI}=$ intervalo de confianza inferior; ICS = intervalo de confianza superior. Elaboración propia mediante el programa estadístico Rstudio. 


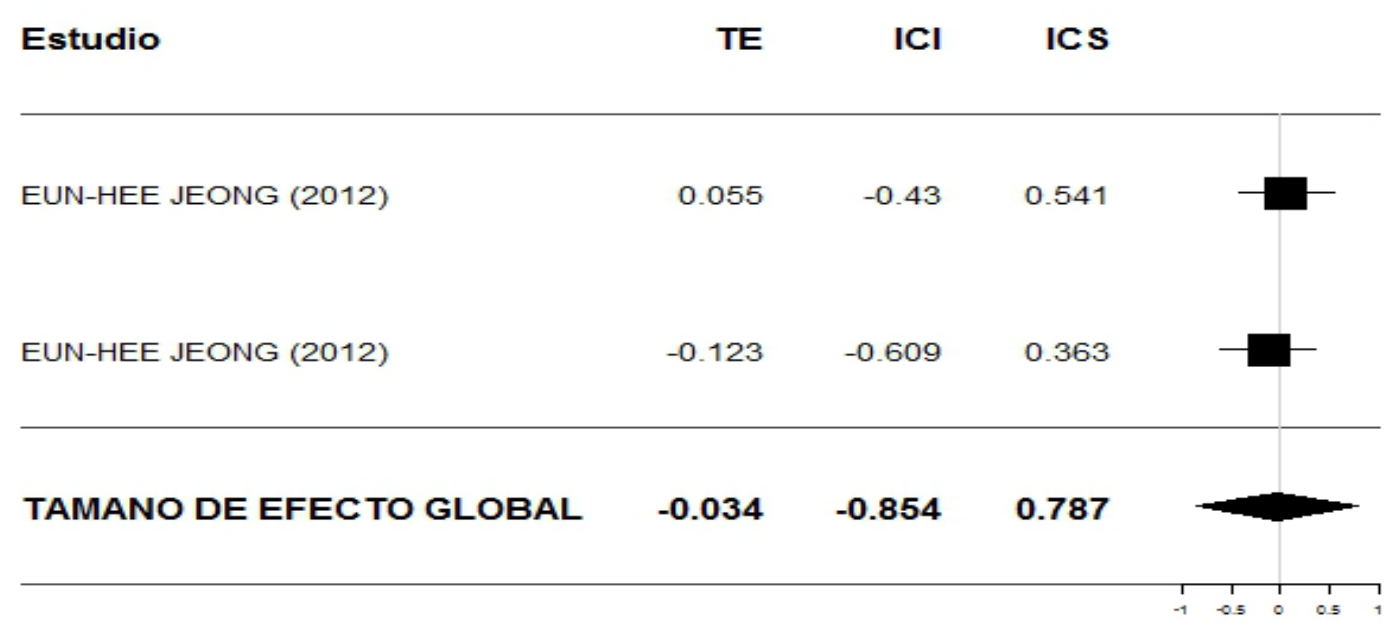

Figura 12. Tamaño de efecto variable rendimiento grupo control; Heterogeneidad $(\mathrm{Q})=.260$ sesgo de publicación $\left(\mathrm{K}_{0}\right)=0$. Con intervalo de confianza al $95 \%$, entra la medición pretest y post test del estado de flow. Nota: TE= Tamaño de efecto; $\mathrm{ICI}=$ Intervalo de confianza inferior; ICS= Intervalo de confianza superior. Elaboración propia mediante el programa estadístico Rstudio.

Correlación estado de flow y rendimiento

Frente a los tamaños de efecto individuales estado de flow y rendimiento, se obtuvo $(\rho=.312$; $p<.05)$ figura $13^{3}$. la interpretación para esta correlación, sugiere que los deportistas que logran presentar flow durante una actividad, es muy probable que aumenten el rendimiento.

3 Frente al outlier que se observa en el gráfico de un TE de 34.6 pertenece a un estudio realizado a futbolistas los cuales presentaron un cambio grande en las mediciones pre test a post test y presentaban una desviación estándar muy baja. Al retirar el outlier y correr de nuevo la correlación, nos arroja $(\rho=.297$; $\mathrm{p}<.05$ ). 


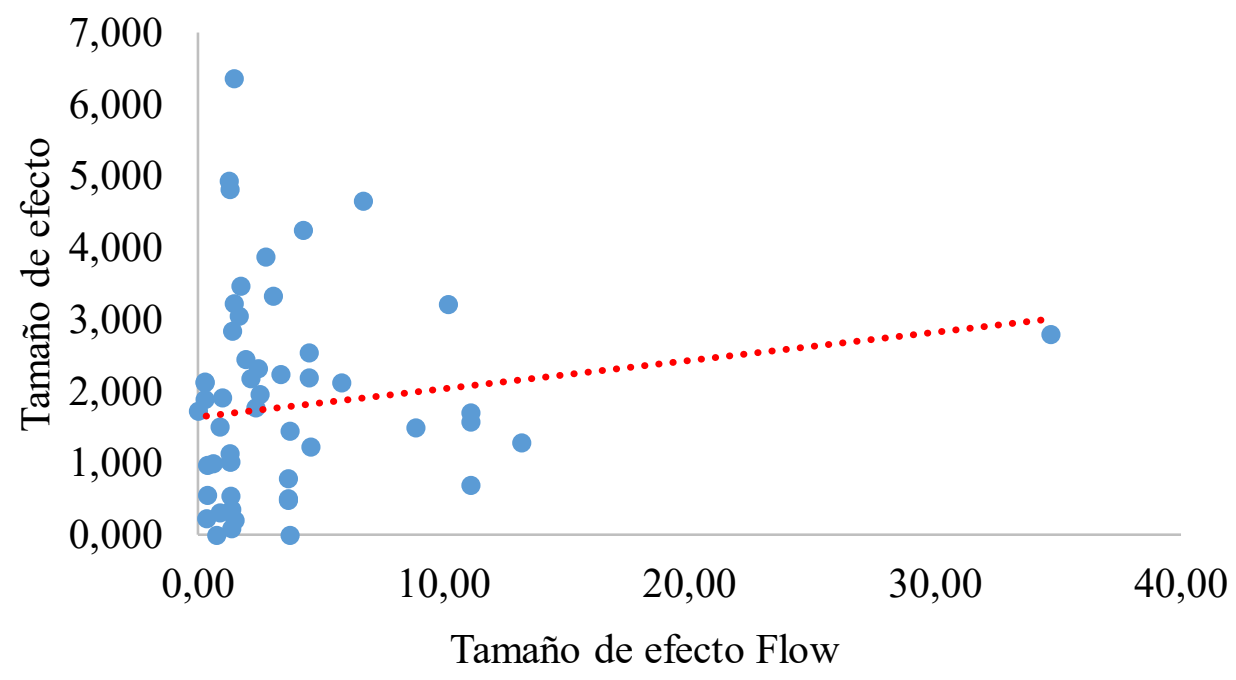

Figura 13. Correlación de los tamaños de efecto del estado de flow. $\rho=.312(p<.05)$. Fuente: elaboración propia.

\section{Variables moderadoras}

Se describe las siete variables moderadoras tanto para estado de flow en la tabla 2 y para rendimiento en la tabla 3. 


\section{Tabla 2.}

Significancia de los TE calculados para estado de Flow.

\begin{tabular}{|c|c|c|c|c|c|c|}
\hline $\begin{array}{c}\text { Variable } \\
\text { moderadora }\end{array}$ & $\mathrm{n}$ & TE & IC & $F$ & $\rho$ & $P$ \\
\hline $\begin{array}{c}\text { Años de } \\
\text { experiencia } \\
\text { deportiva }\end{array}$ & 44 & $1.196^{*}$ & 0.5 a 2.29 & & .140 & .366 \\
\hline Sexo ${ }^{\text {Edad }}$ & 51 & $.773^{*}$ & $0.40 \mathrm{a} 1.62$ & .373 & .204 & $\begin{array}{l}.150 \\
.691\end{array}$ \\
\hline $\begin{array}{l}\text { Hombre } \\
\text { Mujer } \\
\text { mixto }\end{array}$ & $\begin{array}{c}33 \\
7 \\
11\end{array}$ & $\begin{array}{c}.256 \\
1.568 \\
1.869 \text { * }\end{array}$ & $\begin{array}{l}-0.14 a 1.14 \\
-3.71 \mathrm{a} 4.78 \\
0.67 a 3.40\end{array}$ & & & \\
\hline Deporte & & & & 1.091 & & .385 \\
\hline $\begin{array}{c}\text { golf } \\
\text { Tenis } \\
\text { ciclismo ruta } \\
\text { futbol } \\
\text { basketball }\end{array}$ & $\begin{array}{l}17 \\
10 \\
2 \\
5 \\
9\end{array}$ & $\begin{array}{r}1.499 \\
2.283 \\
.833 \\
1.888 \\
.280\end{array}$ & $\begin{array}{l}-2.08 a 4.14 \\
-3.40 a 5.63 \\
-7.46 a 4.86 \\
-4.96 a 5.55 \\
-0.03 a 1.06\end{array}$ & & & \\
\hline $\begin{array}{l}\text { netball } \\
\text { bailarín danza } \\
\text { bádminton }\end{array}$ & $\begin{array}{l}3 \\
2 \\
4\end{array}$ & $\begin{array}{l}1.175 \\
.669 \\
1.390\end{array}$ & $\begin{array}{l}-5.99 a 5.67 \\
-0.65 a 2.33 \\
-5.80 a 5.14\end{array}$ & & & \\
\hline $\begin{array}{l}\text { Tipo } \\
\text { intervención }\end{array}$ & & & & .416 & & .662 \\
\hline $\begin{array}{l}\text { imaginería } \\
\text { hipnosis }\end{array}$ & $\begin{array}{l}25 \\
23\end{array}$ & $\begin{array}{c}1.614^{*} \\
.251\end{array}$ & $\begin{array}{c}0.66 a 2.97 \\
-0.15 a 1.14\end{array}$ & & & \\
\hline $\begin{array}{l}\text { Medición de } \\
\text { Flow }\end{array}$ & & & & .225 & & .799 \\
\hline $\begin{array}{c}\text { (Jackson \& } \\
\text { Marsh, 1996) }\end{array}$ & 33 & .379 & $-0.09 a 1.34$ & & & \\
\hline $\begin{array}{c}\text { (Jackson \& } \\
\text { Eklund, 2002) }\end{array}$ & 17 & $2.066^{*}$ & $0.59 a 3.76$ & & & \\
\hline $\begin{array}{c}\text { (FSS-2; } \\
\text { Jackson \& } \\
\text { Eklund, 2004) }\end{array}$ & 2 & $1.147^{*}$ & $0.71 \mathrm{a} 2.06$ & & & \\
\hline
\end{tabular}

Nota: $T E=$ tamaño de efecto global; IC = intervalo de confianza, $\rho=$ rho de Spearman; $p=$ significancia. Fuente: elaboración propia. 


\section{Tabla 3.}

Significancia de los TE calculados para rendimiento.

\begin{tabular}{|c|c|c|c|c|c|c|}
\hline $\begin{array}{c}\text { Variable } \\
\text { moderadora }\end{array}$ & $\mathrm{n}$ & TE & IC & $\mathrm{F}$ & $\rho$ & $p$ \\
\hline $\begin{array}{c}\text { Años de } \\
\text { experiencia } \\
\text { deportiva }\end{array}$ & 53 & $.361^{*}$ & 0.14 a 1.02 & & -.119 & .397 \\
\hline Sexo & 60 & $.339^{*}$ & 0.13 a 0.96 & 10.185 & -.055 & $\begin{array}{l}.679 \\
.000^{* *}\end{array}$ \\
\hline $\begin{array}{l}\text { Hombre } \\
\text { Mujer } \\
\text { mixto }\end{array}$ & $\begin{array}{c}37 \\
7 \\
16\end{array}$ & $\begin{array}{c}.109 \\
2.600 \\
.412^{*}\end{array}$ & $\begin{array}{l}-0.29 \text { a } 0.99 \\
-5.70 \text { a } 6.63 \\
0.18 \text { a } 1.08\end{array}$ & & & \\
\hline Deporte & & & & 1.697 & & .130 \\
\hline $\begin{array}{c}\text { golf } \\
\text { Tenis } \\
\text { ciclismo ruta } \\
\text { futbol } \\
\text { basketball }\end{array}$ & $\begin{array}{l}17 \\
18 \\
2 \\
5 \\
9\end{array}$ & $\begin{array}{l}1.335 \\
.90{ }^{*} \\
.818 \\
1.123 \\
.134\end{array}$ & $\begin{array}{l}-1.76 a 3.80 \\
0.47 \text { a } 1.83 \\
-8.25 a 5.03 \\
-4.70 \text { a } 4.50 \\
-0.17 a 0.91\end{array}$ & & & \\
\hline netball & 3 & 1.891 & $-5.84 a 5.78$ & & & \\
\hline $\begin{array}{l}\text { bailarín danza } \\
\text { bádminton }\end{array}$ & $\begin{array}{l}2 \\
4\end{array}$ & $\begin{array}{l}.216 \\
3.009\end{array}$ & $\begin{array}{l}-0.11 \text { a } 1.02 \\
-5.54 a 7.10\end{array}$ & & & \\
\hline Tipo intervención & & & & 4.175 & & $.020^{*}$ \\
\hline $\begin{array}{l}\text { imaginería } \\
\text { hipnosis }\end{array}$ & $\begin{array}{l}34 \\
23\end{array}$ & $\begin{array}{l}.419^{*} \\
.092\end{array}$ & $\begin{array}{r}0.18 \text { a1.09 } \\
-0.31 \text { a0.98 }\end{array}$ & & & \\
\hline $\begin{array}{l}\text { Medición } \\
\text { Rendimiento }\end{array}$ & & & & 1.394 & & .248 \\
\hline $\begin{array}{l}\text { Rendimiento } \\
\text { pruebas deportivas } \\
\text { Rendimiento }\end{array}$ & 21 & 1.799 & -1.20 a4.22 & & & \\
\hline $\begin{array}{l}\text { pruebas motoras } \\
\text { Rendimiento }\end{array}$ & 30 & $.397^{*}$ & 0.14 a1.10 & & & \\
\hline $\begin{array}{c}\text { subjetivo } \\
\text { entrenador } \\
\text { Rendimiento }\end{array}$ & 2 & .205 & $-0.13 a 1.02$ & & & \\
\hline $\begin{array}{l}\text { subjetivo del } \\
\text { deportista }\end{array}$ & 6 & .092 & $-0.37 a 1.05$ & & & \\
\hline $\begin{array}{l}\text { Resultado en } \\
\text { competencia }\end{array}$ & 2 & .818 & $-8.25 a 5.03$ & & & \\
\hline
\end{tabular}

Nota: $T E=$ tamaño de efecto global; $I C=$ intervalo de confianza, $\rho=$ rho de Spearman; $p=$ significancia. Fuente: elaboración propia. 
Años de experiencia deportiva

Los años de experiencia expuestos en los diferentes estudios metaanalizados se encuentran en un rango de 2 a 15 años de experiencia deportiva.

Estado de flow: se observa una tendencia levemente ascendente a casi nula a medida que se aumentan los años de experiencia en los tamaños de efecto individuales. De los 45 tamaños de efecto estado de flow el mayor porcentaje se centra entre los 4 y 8 años de experiencia. Se establece una correlación positiva muy baja, no siendo estadísticamente significativa $(\rho=.140$; $p=.366)$. Los años de experiencia no constituyen para esta muestra un factor moderador de relación significativa del tamaño de efecto en la figura 14, en las intervenciones psicológicas de técnicas de preparación mental basadas en imaginería o hipnosis sobre el estado flow 4 .

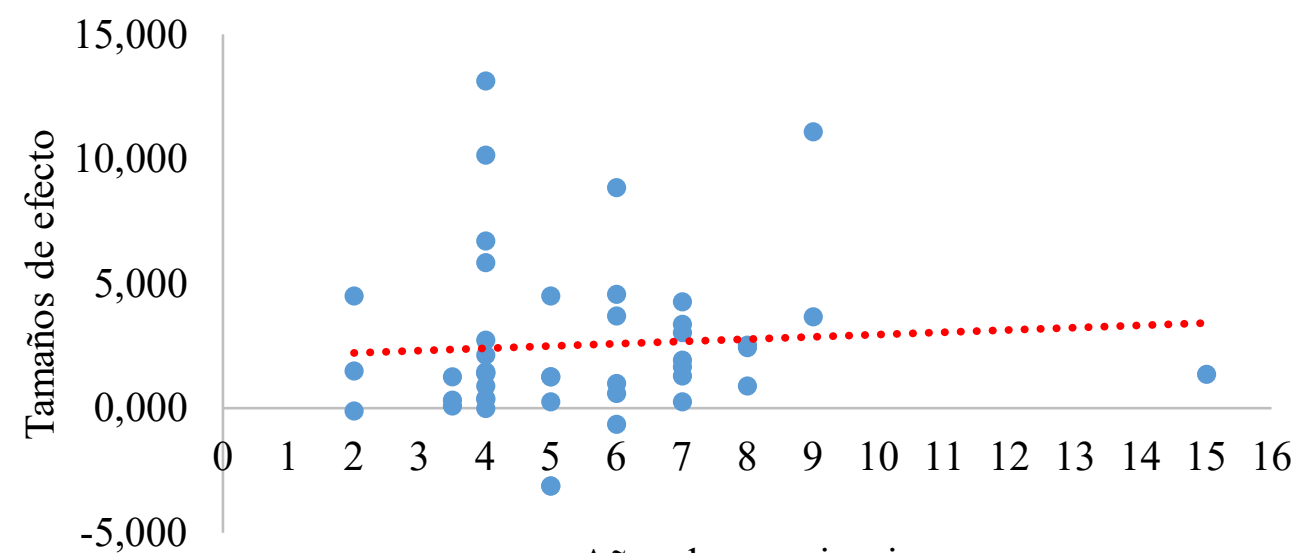

Años de experiencia

Figura 14. Correlación de los tamaños de efecto del estado de Flow y años de experiencia deportiva, $\rho=.140(p=.366)$. Fuente: elaboración propia.

Rendimiento: se observa una tendencia negativa a medida que se aumentan los años de experiencia y el rendimiento deportivo de los diferentes tamaños de efecto individuales. De los 55 tamaños de efectos de rendimiento el mayor porcentaje se centra entre los 4 y 7 años de

\footnotetext{
${ }^{4}$ Se presentan outliers, un total de seis. Al retirarlos se obtiene $(\rho=.177 ; p=.288)$.
} 
experiencia. Se establece una correlación negativa y no significativa $(\rho=-.199 ; p=.397)$. Los años de experiencia no constituyen para esta muestra un factor moderador de relación significativa del tamaño de efecto en la figura 15, en intervenciones psicológicas de técnicas de preparación mental basadas en imaginería o hipnosis sobre el rendimiento en actividades deportivas ${ }^{5}$.

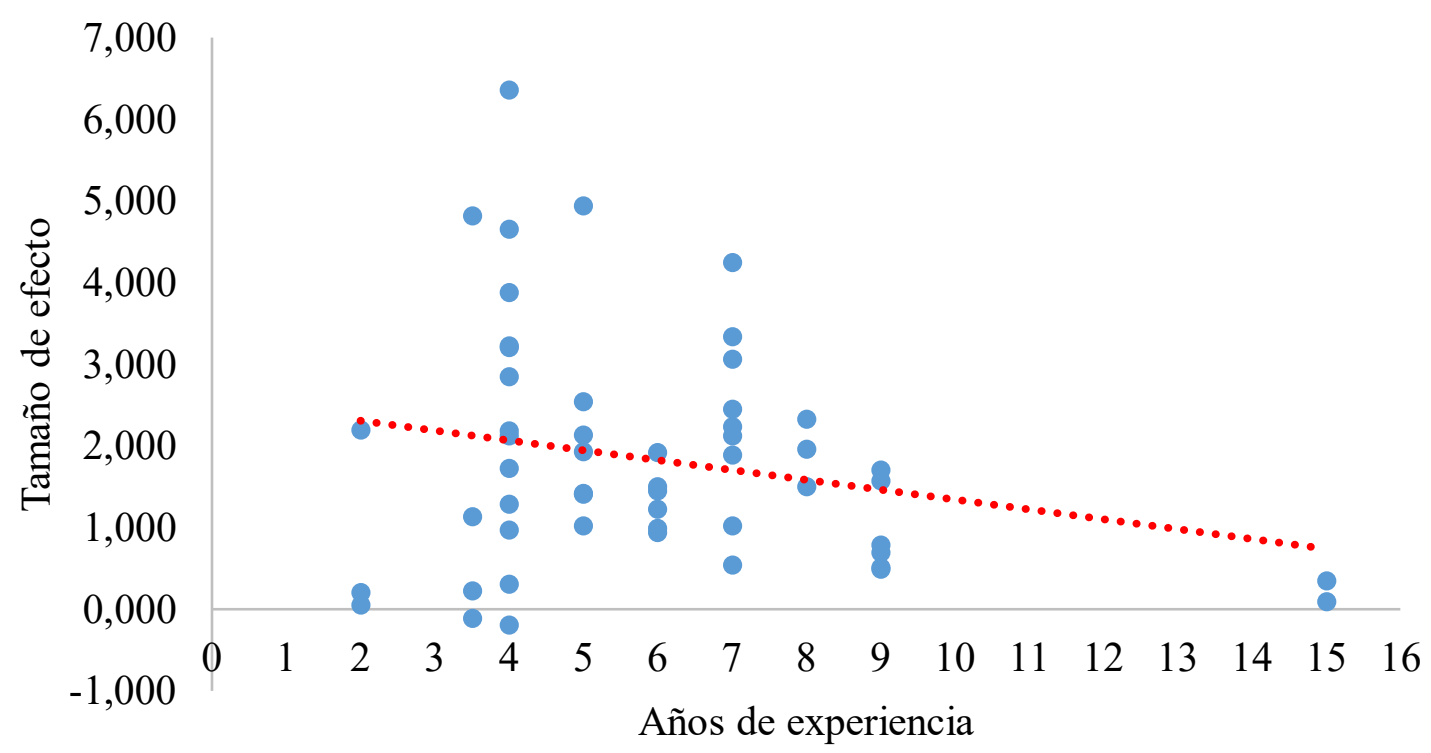

Figura 15. Correlación de los tamaños de efecto de rendimiento y años de experiencia deportiva, $\rho=.199(p=.397)$. Fuente: elaboración propia.

\section{Edad}

La edad de los diferentes sujetos que se encuentran en los estudios metaanalizados, se presenta entre los 13 a los 52 años.

Estado de flow: se observa una tendencia positiva entre la edad y estado de flow de los diferentes tamaños de efectos individuales. De los 52 tamaños de efecto estado de flow, el mayor porcentaje se centra entre los 20 y 25 años. Se establece una correlación positiva pero

\footnotetext{
${ }^{5}$ Se presentan outliers, un total de siete. Al retirarlos se obtiene $(\rho=.036 ; p=.815)$.
} 
no significativa $(\rho=.204 ; p=.150)$. La edad no constituye para esta muestra un factor moderador de relación significativa del tamaño de efecto en la figura 16, en intervenciones psicológicas de técnicas de preparación mental basadas en imaginería o hipnosis sobre el estado de flow ${ }^{6}$.

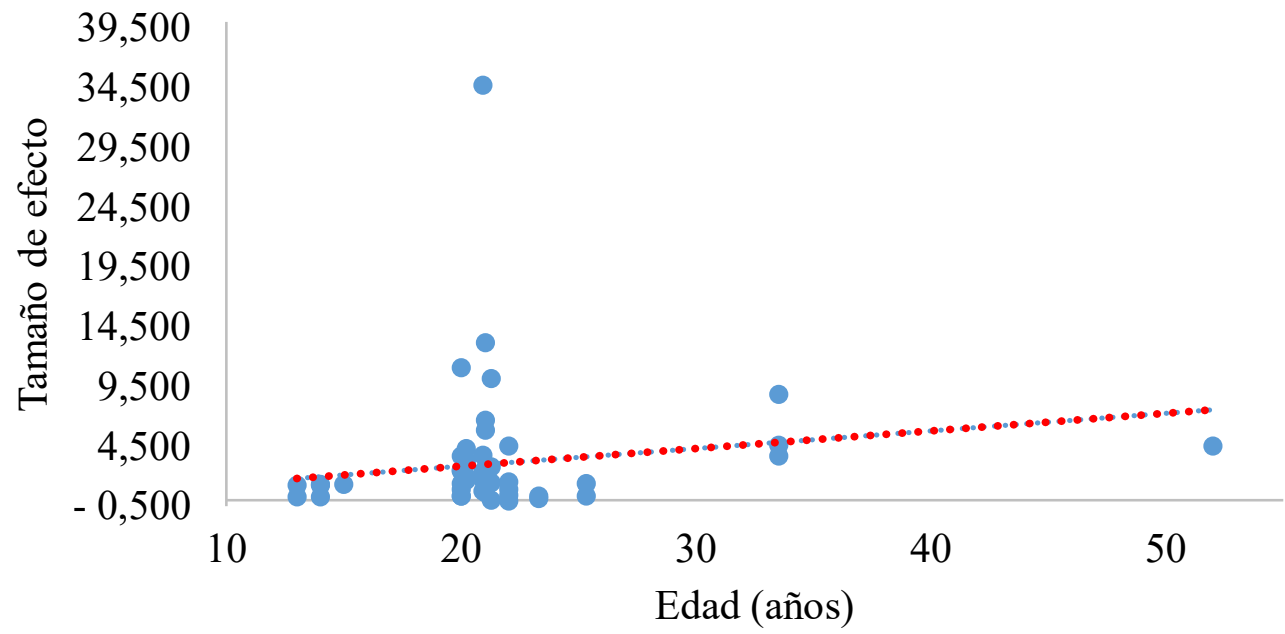

Figura 16. Correlación de los tamaños de efecto del estado de flow y la edad. $. \rho=.204$ $(p=.150)$. Fuente: elaboración propia.

Rendimiento: se observa una tendencia negativa entre la edad y el rendimiento de los diferentes tamaños de efecto individuales. De los 62 tamaños de efectos de rendimiento, el mayor porcentaje se centra entre los 20 y 22 años. Se establece una correlación negativa y no significativa $(\rho=-.055 ; p=.679)$. La edad no constituye para esta muestra un factor moderador de relación significativa del tamaño de efecto en la figura 17, en intervenciones psicológicas de técnicas de preparación mental basadas en imaginería o hipnosis sobre el rendimiento de actividades deportivas ${ }^{7}$.

\footnotetext{
${ }^{6}$ Se presentan outliers, un total de cinco. Al retirarlos se obtiene $(\rho=.050 ; p=.740)$.

7 Se presentan outliers, un total de cinco. Al retirarlos se obtiene $(\rho=-.107 ; p=.437)$.
} 


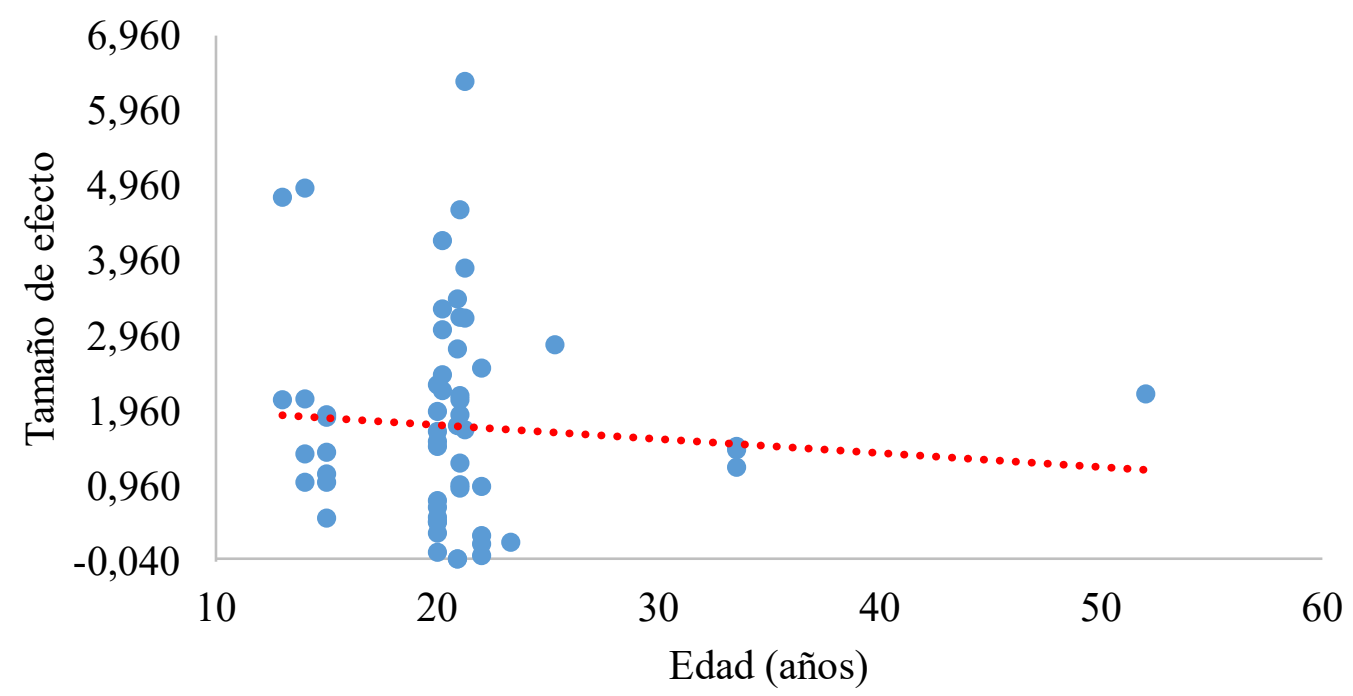

Figura 17. Correlación de los tamaños de efecto de rendimiento y la edad. $\rho=-.055(p=.679)$. Fuente: elaboración propia.

Sexo

Estado de flow: para la clasificación hombres ( $\mathrm{TE}=.256 ; p=.214 ; \mathrm{n}=33$ ), para las mujeres (TE= $1.568 ; p=.561 ; \mathrm{n}=7)$ y la clasificación mixta $(\mathrm{TE}=1.869 ; p<.05 ; \mathrm{n}=11)$. El sexo clasificado individualmente tanto en hombres como en mujeres, no son un factor influyente ni significativo en los efectos del estado de flow; pero contemplado desde la clasificación mixta si es un factor influyente y significativo en los efectos del estado de flow con intervenciones psicológicas de técnicas de preparación mental basadas en imaginería o hipnosis descrito en la figura 18. Se establece que no hay diferencias significativas entre las clasificaciones de la variable sexo $(p=.691)$. 


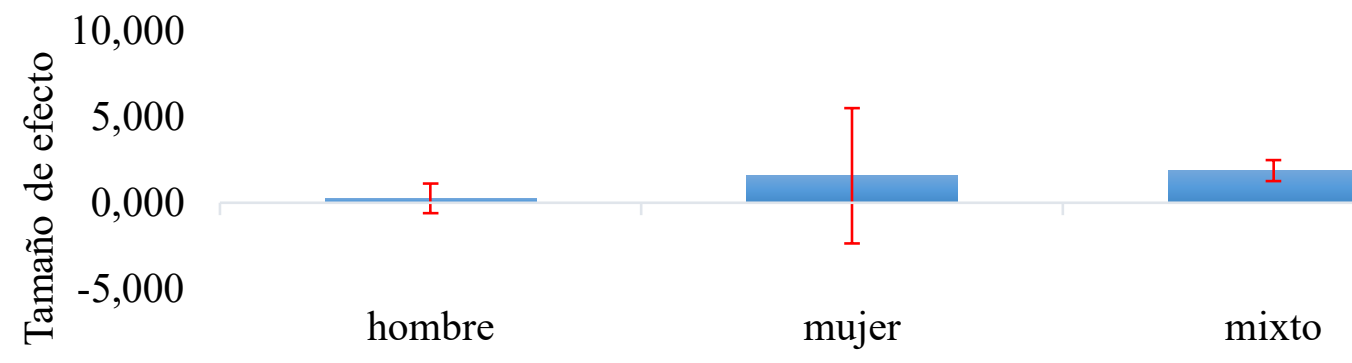

Sexo

Figura 18. Tamaño de efecto según el sexo de los participantes en los estudios sobre el estado de flow, $F(2,49)=.373(p=.691)$. Fuente: elaboración propia.

Rendimiento: para la clasificación hombres ( $\mathrm{TE}=.109 ; p=.594$; $\mathrm{n}=37$ ), para las mujeres (TE= $2.600 ; p=.54 ; \mathrm{n}=7)$ y la clasificación mixta $(\mathrm{TE}=.412 ; p<.05 ; \mathrm{n}=16)$. El sexo clasificado individualmente tanto en hombres como en mujeres no es un factor influyente ni significativo en los efectos del rendimiento; pero contemplado desde la clasificación mixta si es un factor influyente y significativo en los efectos del rendimiento con intervenciones psicológicas de técnicas de preparación mental basadas en imaginería o hipnosis se describe en la figura 19. Si hay diferencias significativas entre las tres clasificaciones $(p<.001)$.

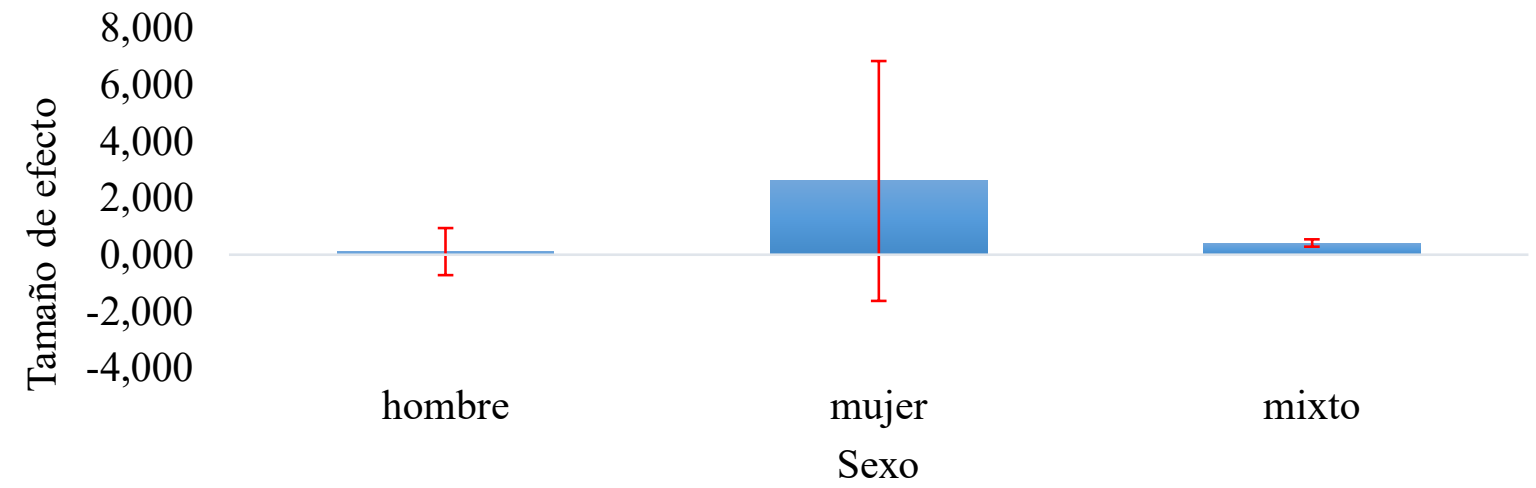

Figura 19. Tamaño de efecto según el sexo de los participantes en los estudios sobre el rendimiento, $F(2,57)=10.185\left({ }^{*} p<.001\right)$. Fuente: elaboración propia. 
Deporte

Se divide el deporte en individuales y de conjunto.

Flow: para deportes individuales $(\mathrm{TE}=1.709 ; p<.001 ; \mathrm{n}=31)$ y para deportes de conjunto $(\mathrm{TE}=$ 0.288; $p=.069 ; \mathrm{n}=21)$. Los deportes individuales son un factor influyente y significativo en los efectos del estado de flow con intervenciones psicológicas de técnicas de preparación mental basadas en imaginería o hipnosis se describe en la figura 20 . Se establece que no hay diferencias significativas entre las dos categorías $(p=.417)$.

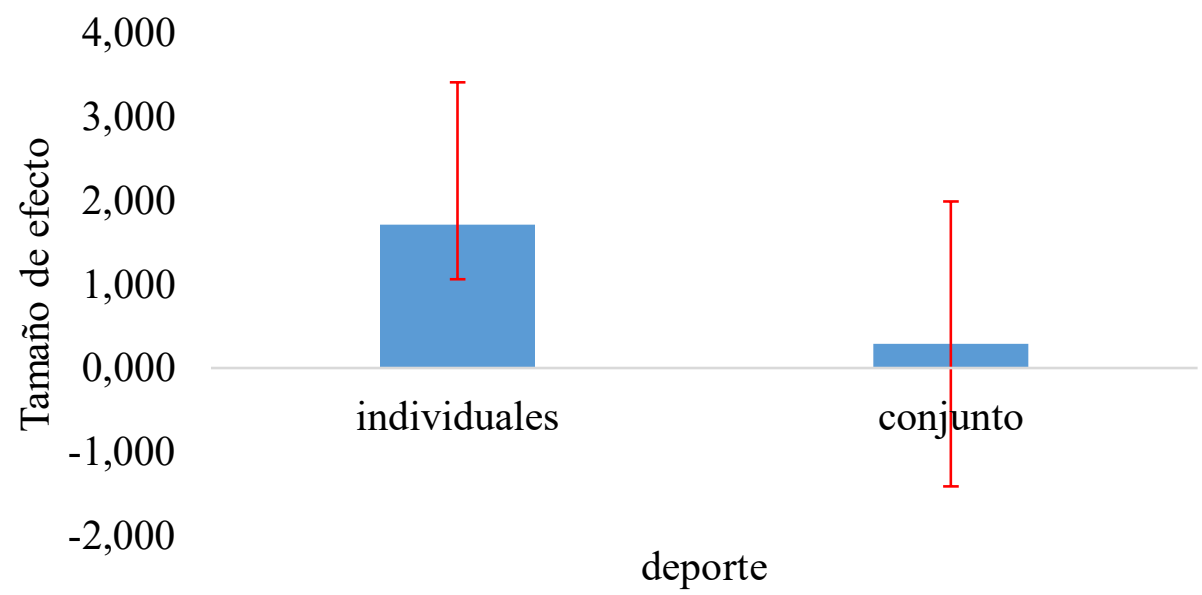

Figura 20. Tamaño de efecto según la categoría deportiva de los estudios que presentan el estado de flow, $F(1,50)=.668(p=.417)$. Fuente: elaboración propia.

Rendimiento: para deportes individuales $(\mathrm{TE}=0.289 ; p<.05 ; \mathrm{n}=38)$ y para deportes de conjunto (TE= 0.143; $p=.366 ; n=22)$. Los deportes individuales son un factor influyente y significativo en los efectos del estado de flow con intervenciones psicológicas de técnicas de preparación mental basadas en imaginería o hipnosis se describe en la figura 21 . Se establece que no hay diferencias significativas entre las dos categorías $(p=.089)$. 


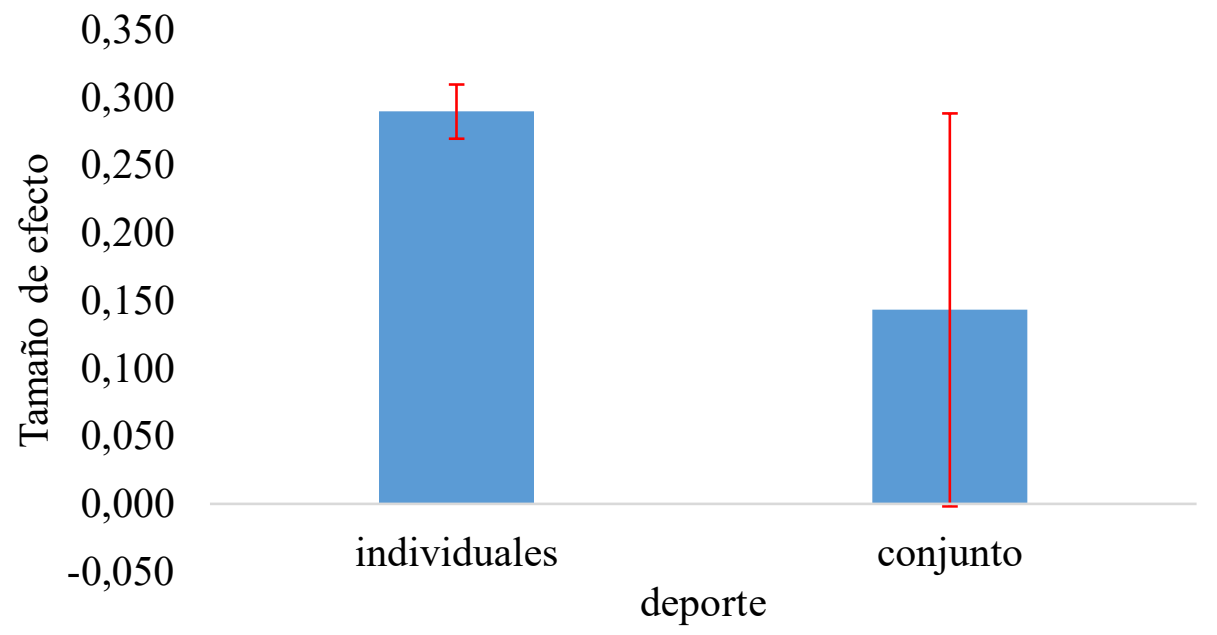

Figura 21. Tamaño de efecto según la categoría deportiva de los estudios que presentan el estado de flow, $F(1,50)=2.979(p=.089)$. Fuente: elaboración propia.

Tipo de intervención

Los tipos de intervención que se meta-analizaron estuvieron definidas como: imaginería e hipnosis.

Estado de flow: para la imaginería se obtuvieron valores de $(\mathrm{TE}=1.614 ; p<.05 ; \mathrm{n}=30)$, para hipnosis $(\mathrm{TE}=.251 ; p=.226 ; \mathrm{n}=22)$. Se concluye que, la imaginería es un factor influyente y significativo en los efectos del estado de flow con intervenciones psicológicas de técnicas de preparación mental se describe en la figura 22. Se establece que no hay diferencias significativas entre los tipos de intervenciones $(p=.841)$. 


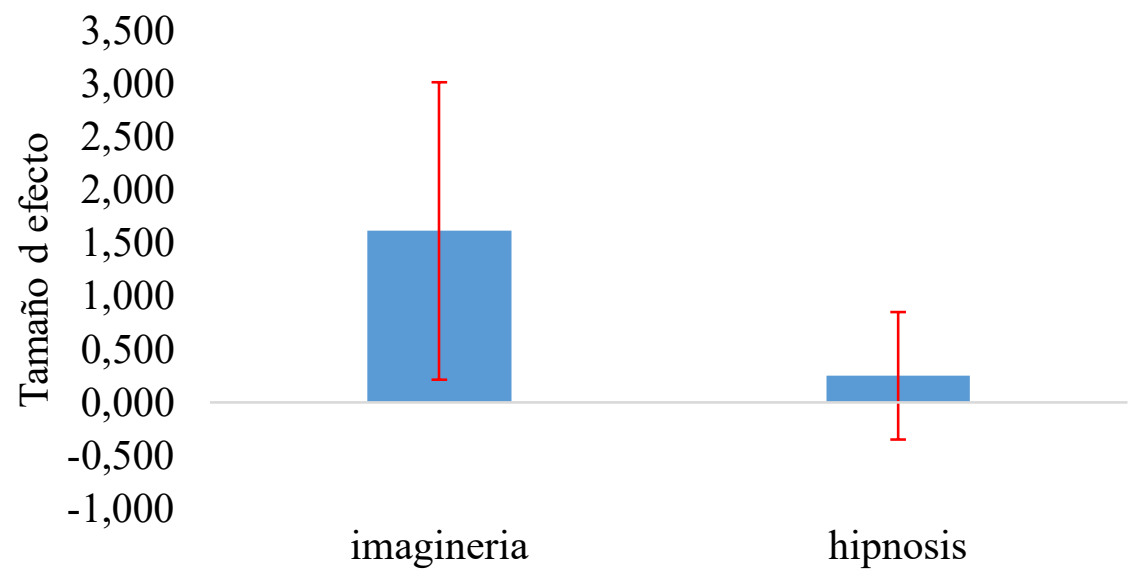

Tipo de intervención

Figura 22. Tamaño de efecto según el tipo de intervención de los estudios sobre estado de flow, $F(1,50)=.416(p=.662)$. Fuente: elaboración propia.

Rendimiento: para la imaginería se obtuvieron valores de $(\mathrm{TE}=0.419 ; p<.001 ; n=34)$, para hipnosis (TE=0.092; $p=.657 ; n=16$ ). Se concluye que, la imaginería es un factor influyente y significativo en los efectos del estado de flow con intervenciones psicológicas de técnicas de preparación mental se describe en la figura 23. Se establece que si hay diferencias significativas entre los tipos de intervenciones $(p<.05)$.

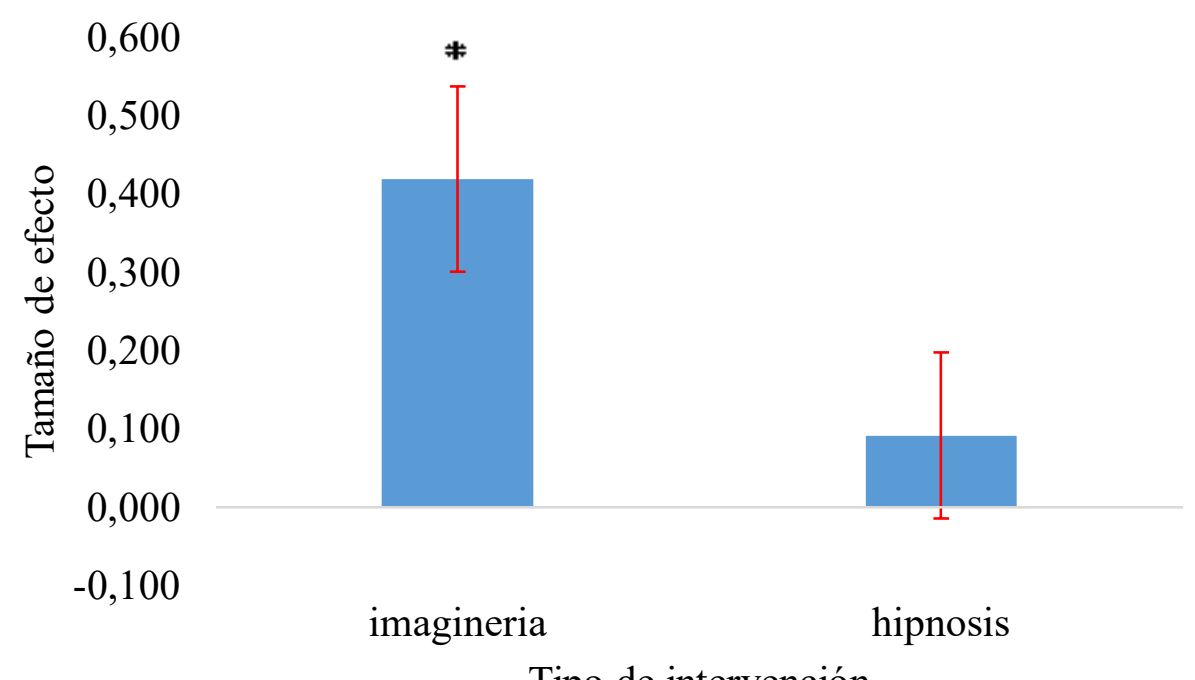

Figura 23. Tamaño de efecto según el tipo de intervención en los estudios sobre el rendimiento, $F(1,58)=7.829\left({ }^{*} p<.05\right)$. Fuente: elaboración propia. 
Medición estado de flow:

Estado de flow: Se evidencia que para la escala de Jackson y Marsh (1996) $(\mathrm{TE}=.379 ; p=.117$; $\mathrm{n}=33$ ), para la escala Jackson y Eklund (2002) $(T E=2.066 ; p<.05 ; n=17)$, Jackson y Eklund (2004) $(T E=1.147 ; p<.001 ; n=2)$. La escala (Jackson y Marsh, 1996), no es un factor influyente ni significativo; pero las escalas (Jackson y Eklund, 2002) y (Jackson y Eklund, 2004), si son un factor influyente y significativo en los efectos del estado de flow con intervenciones psicológicas de técnicas de preparación mental basadas en imaginería o hipnosis se describe en la figura 24. Se establece que no hay diferencias significativas entre las escalas $(p=.799)$.

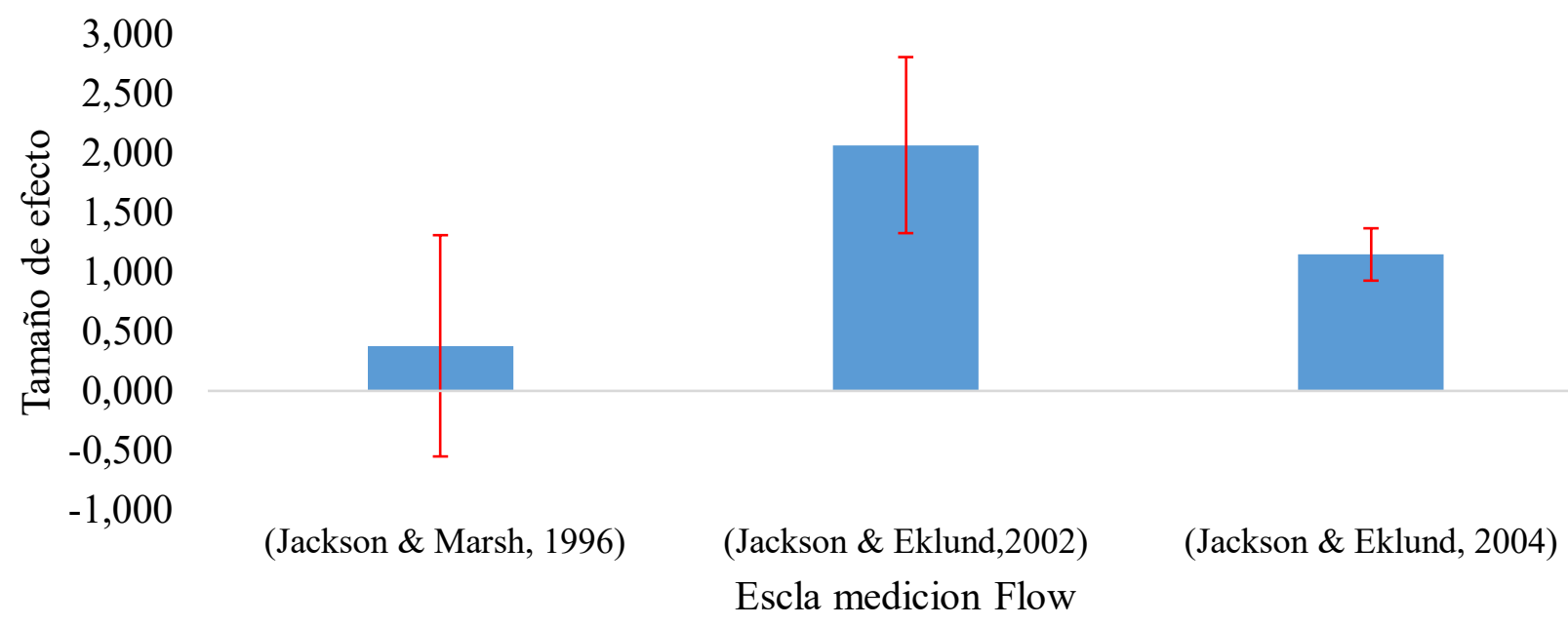

Figura 24. Tamaño de efecto según la escala de medición estado de flow de los estudio, $\mathrm{F}$ $(2,49)=.225(p=.799)$. Fuente: elaboración propia.

Medición rendimiento:

Rendimiento: para el rendimiento en pruebas deportivas se obtuvo (TE=1.799; $p=.240 ; n=21$, en pruebas motoras $(\mathrm{TE}=.397 ; p<.05 ; \mathrm{n}=30)$, para el rendimiento subjetivo entrenador $(\mathrm{TE}=$ $.205 ; p=.235 ; \mathrm{n}=2)$, para el rendimiento subjetivo del deportista $(\mathrm{TE}=.092 ; p=.702 ; \mathrm{n}=6) \mathrm{y}$ resultado en competencia $(\mathrm{TE}=.818 ; p=.860 ; \mathrm{n}=2)$. El rendimiento en pruebas deportivas, rendimiento subjetivo entrenador, rendimiento subjetivo del deportista y resultado en 
competencia, no son un factor influyente ni significativo del TE; por el contrario, el rendimiento en pruebas motoras si es un factor influyente y significativo del TE de intervenciones psicológicas con técnicas de preparación mental basadas en imaginería o hipnosis se describe en la figura 25 . Se establece que no hay diferencias significativas entre las clasificaciones de rendimiento $(p=.248)$.

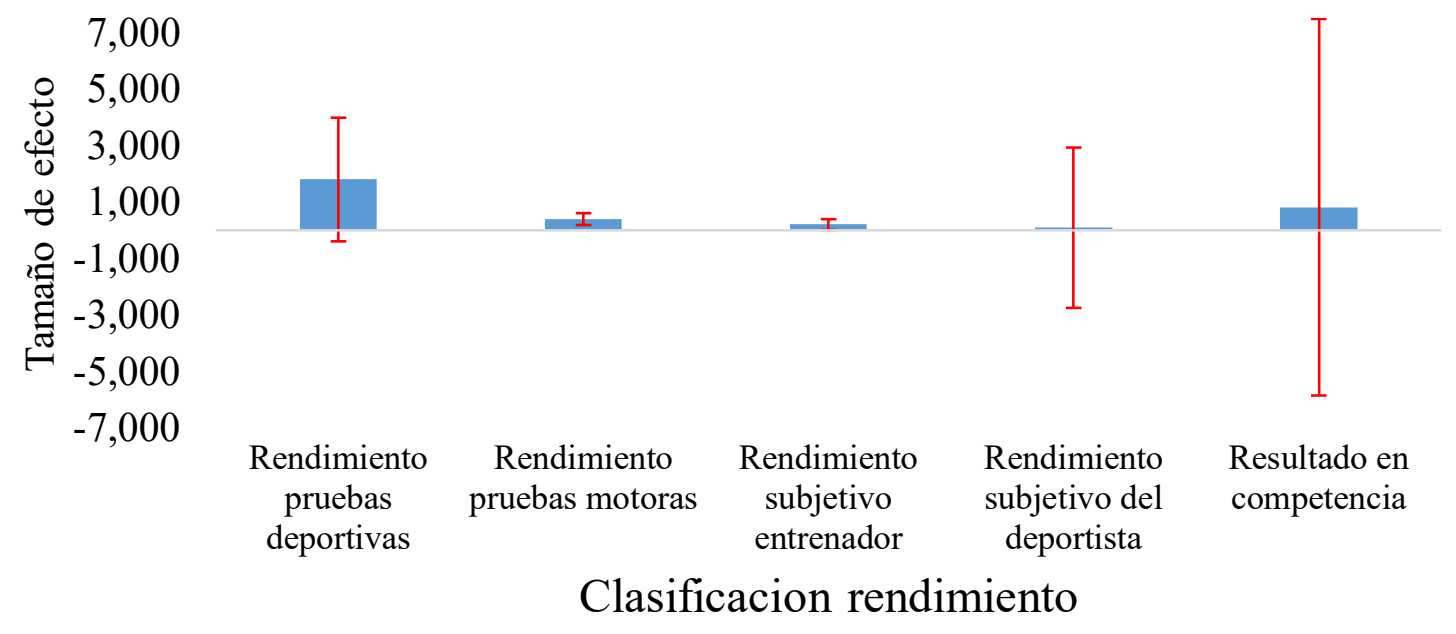

Figura 25. Tamaño de efecto según la clasificación de rendimiento los estudio, $F(4,55)=1.394$ $(p=.248)$. Fuente: elaboración propia. 


\section{Discusión}

Se planteó como objetivo principal, dilucidar el efecto de técnicas de preparación mental cognitiva sobre el estado de flow y el rendimiento deportivo, por medio del diseño meta analítico intragrupo y la búsqueda de un tamaño de efecto global modelo de efectos fijos. Seguido se realizó una correlación entre las dos variables dependientes y se buscó identificar cuales factores influyen en los resultados obtenidos, por lo que se llevó a cabo un análisis de variables moderadoras.

\section{Tamaño de efecto global}

Se evidencia una mejora significativa tanto en el estado de flow como en el rendimiento deportivo, mediante las técnicas de preparación mental cognitivas o afectivas específicamente con la imaginería. Esto ratifica lo concluido por Koehn, Morris y Watt (2014a), que en técnicas con un corte de preparación mental cognitiva como la imaginería se obtuvo una mejora significativa sobre el estado de flow he igualmente sobre el rendimiento desde una línea pre a post. La correlación positiva aunque moderada que señala los resultados obtenidos, enmarcan lo expuesto por (Jackson, Thomas, Marsh y Smethurst, 2001) donde evidencia la relación que existe entre el constructo de estado de flow y el rendimiento deportivo. Asimismo, siguiendo lo expuesto por Csikszentmihalyi $(\underline{1975})$ con referencia a flow, quien pronosticaba el objetivo fundamental de entrar en esta zona de satisfacción plena y los resultados positivos exteriores que se lograban.

\section{Variables moderadoras}

Flow: el estado de flow no es propiamente caracterizado por el género y esto se evidencia con lo expuesto por (Jackson, 1992, 1995; Jackson, et al., 1998; Koehn, 2007), tanto para hombres como para mujeres los efectos del estado de flow, se presenta en similares condiciones. Ahora bien, en cuanto a las técnicas psicológicas que mayor efecto tienen sobre las mejoras en el 
estado de flow arroja como resultado lo expuesto por autores como (Casby y Moran, 1998; Jeong, 2012; Koehn y Díaz-Ocejo, 2016; Nicholls, Polman y Holt, 2005; Pain et al., 2011; Sardon, Mazaulan, y Mohamed, 2016; Welo, 2009), quienes concluyen y recomiendan la utilización de la imaginería como mecanismo para incrementar el estado de flow en deportistas, siendo la técnica psicológica de mayor interés de investigación y donde se han evidenciado resultados relevantes para beneficiar el estado de flow durante una actividad deportiva. Contrario a los resultados obtenidos con intervenciones de hipnosis, las cuales no han evidenciado una clara posición en la afectación que pueda tener sobre el estado de flow. Posición que se mantiene en fundamento, al TE descrito en este metaanálisis al no ser estadísticamente significativo.

Rendimiento: investigaciones que evalúan el rendimiento presentado en una actividad deportiva, después de haber realizado una intervención de imaginería o hipnosis (Nicholls et al., 2005; Pates, Cowen y Karageorghis, 2012; Welo, 2009), han evidenciado un aumento significativo del rendimiento destacando estos resultados en deportes individuales. Los resultados aquí expuestos, resaltan que en la variable dependiente de rendimiento, la categoría de deportes individuales presenta un efecto moderador del tamaño de efecto global; diversos estudios (Koehn, 2007; Koehn et al., 2014b; Sardon et al., 2016) destacan en la variable rendimiento un aumento significativo en tenis. Frente a las técnicas de preparación mental para incrementar el rendimiento deportivo se encuentra la imaginería, igualmente expuesto en estudios de (Casby y Moran, 1998; Koehn y Díaz-Ocejo, 2016; Koehn et al., 2014b; Nicholls et al., 2005) y que presenta mejorías significativas en la variable de medición del rendimiento en diversas disciplinas deportivas. Frente a los estudios meta analizados se observa que un factor moderador e influyente frente a los resultados del tamaño de efecto global establecido para el rendimiento en cuanto al tipo de medición, es la medición de pruebas motoras, esto se expone en evidencia en diversos estudios que así utilizan este tipo de medición del rendimiento 
(Nicholls et al., 2005; Pates, Karageorghis, Fryer y Maynard, 2003; Pates y Maynard, 2000), asimismo, realizando recomendaciones de que este tipo de evaluación es la más efectiva para observar las mejoras en el rendimiento de una tarea específica que se pueda observar en una tarea.

\section{Limitaciones}

Este metaanálisis no se encuentra exento de limitaciones. Es de resaltar que la falta de estudios realizados con un diseño experimental puro limita la generalización de algunas conclusiones que se puedan sacar, al arrojar supuestos de generalización con estudios de casos individuales y con características particulares. Para la variable dependiente de estado de flow sería de gran importancia contar con un puntaje para cada una de las dimensiones que componen este constructo, para así poder analizar cada dimensión como una variable moderadora y buscar su influencia sobre el puntaje de flow global establecido por las escalas que se implementan en cada estudio.

Implicaciones del estado de flow y el rendimiento en campo

Flow como un estado psicológico ideal para un deportista, proporciona elementos a nivel psicológico para el deportista, que logran un rendimiento durante I actividad. Por tal motivo, es de vital importancia que deportistas y entrenadores, logren establecer estrategias para encontrar el estado de flow durante la actividad. La imaginería como un camino a buscar este estado ideal, es la manera más adecuada para lograrlo. 


\section{Conclusión}

Técnicas psicológicas como hipnosis e imaginería, presentan beneficios, así lo contemplan los resultados que brinda este metaanálisis. De igual, se resalta que presentar un estado de flow durante una actividad deportiva, ayudaría a tener un aumento en el rendimiento durante la actividad; asimismo, variables como los años de experiencia deportiva juegan un papel relevante a la hora de presentar flow durante una actividad. Se podría considerar tomar estos resultados para realizar estudios experimentales de un diseño experimental puro en deportes tanto de conjunto y/o individual, evidenciando las diferencias que puedan existir y analizar el estado de flow desde una perspectiva multidimensional, tomando el resultado de cada una de las nueve dimensiones del estado de flow para determinar cual tiene mayor influencia en esta relación aquí presentada. 


\section{Referencias}

Borenstein, M., Hedges, L. V., Higgins, J. P. T., y Rothstein, H. R. (2011). Introduction to MetaAnalysis. John Wiley \& Sons.

Cabrera Macías, Y., López González, E. J., Ramos Rangel, Y., González Brito, M., Valladares González, A. M., y López Angulo, L. (2013). La hipnosis: Una técnica al servicio de la Psicología. MediSur, 11(5), 534-541. Recuperado de http://scielo.sld.cu/pdf/ms/v11n5/ms08511.pdf

Casby, A., y Moran, A. (1998). Exploring mental imagery in swimmers: A single-case study design. The Irish Journal of Psychology, 19(4), 525-531. doi: https://doi.org/10.1080/03033910.1998.10558212

Csikszentmihalyi, M. (1975). Beyond boredom and anxiety: experiencing flow in work and play. California, Estados Unidos: A Wiley Company. Recuperado de https://www.amazon.com/Beyond-Boredom-Anxiety-Experiencing-Flow/dp/0787951404

Jackson, S. A. (1992). Athletes in flow: A qualitative investigation of flow states in elite figure skaters. Journal of applied sport psychology, 4(2), 161-180. doi: https://doi.org/10.1080/10413209208406459

Jackson, S. A. (1995). Factors influencing the occurrence of flow state in elite athletes. Journal of Applied Sport Psychology, 7(2), 138-166. doi: https://doi.org/10.1080/10413209508406962

Jackson, S. A., y Eklund, R. C. (2002). Assessing flow in physical activity: the flow state scale-2 and dispositional flow scale-2. Journal of Sport \& Exercise Psychology, 24(2). doi: https://doi.org/10.1123/jsep.24.2.133 
Jackson, S. A., y Eklund, R. C. (2004). The Flow Scales Manual. Morgantown, USA: Fitness Information Technology. Recuperado de https://www.amazon.com/-les/SusanJackson/dp/1885693516

Jackson, S. A., Ford, S. K., Kimiecik, J. C., y Marsh, H. W. (1998). Psychological correlates of flow in sport. Journal of Sport and exercise Psychology, 20(4), 358-378. doi: https://doi.org/10.1123/jsep.20.4.358

Jackson, S. A., y Marsh, H. W. (1996). Development and validation of a scale to measure optimal experience: The Flow State Scale. Journal of Sport and Exercise Psychology, 18(1), 17-35. Recuperado de de https://pdfs.semanticscholar.org/553c/9421dbb9d23735b0936e3743eaa021f0b4f4.pdf

Jackson, S. A., Martin, A. J., y Eklund, R. C. (2008). Long and short measures of flow: The construct validity of the FSS-2, DFS-2, and new brief counterparts. Journal of Sport and Exercise Psychology, 30(5), 561-587. doi: https://doi.org/10.1123/jsep.30.5.561

Jackson, S. A., Thomas, P. R., Marsh, H. W., y Smethurst, C. J. (2001). Relationships between flow, self-concept, psychological skills, and performance. Journal of Applied Sport Psychology, 13(2), 129-153. doi: https://doi.org/10.1080/104132001753149865

Jeong, E.H. (2012). The application of imagery to enhance "flow state" in dancers (Tesis doctorado). Victoria University. Recuperado de https://core.ac.uk/download/pdf/10836166.pdf

Koehn, S. (2007). Propensity and attainment of flow state (Tesis doctorado). Victoria University. Recuperado de https://core.ac.uk/download/pdf/10827156.pdf

Koehn, S., y Díaz-Ocejo, J. (2016). Imagery intervention to increase flow state: A single-case study with middle-distance runners in the state of Qatar. International Journal of Sport and Exercise Psychology, 1-14. doi: https://doi.org/10.1080/1612197X.2016.1187653 
Koehn, S., Morris, T., y Watt, A. P. (2014a). Imagery Intervention to Increase Flow State and Performance in Competition. The Sport Psychologist, 28(1), 48-59. doi: https://doi.org/10.1123/tsp.2012-0106

Koehn, S., Morris, T., y Watt, A. P. (2014b). Imagery Intervention to Increase Flow State and Performance in Competition. The Sport Psychologist, 28(1), 48-59. doi: https://doi.org/10.1123/tsp.2012-0106

Lindsay, P., Maynard, I., y Thomas, O. (2005). Effects of Hypnosis on Flow States and Cycling Performance. The Sport Psychologist, 19(2), 164-177. doi: https://doi.org/10.1123/tsp.19.2.164

Montgomery, G. H., y Schnur, J. B. (2005). Eficacia y aplicación de la hipnosis clínica. Papeles del Psicólogo, 25(89), 3-8. Recuperado de https://www.redalyc.org/pdf/778/77808902.pdf

Nakamura, J., y Csikszentmihalyi, M. (2002). Oxford Handbook of positive psycholog. En: C.R. Snyder y S.J. Lopez (Eds.), The concept of flow (pp.89-105). Estados Unidos: Oxford University Press. Recuperado de http://eweaver.myweb.usf.edu/2002-Flow.pdf

Nicholls, A., Polman, R., y Holt, N. (2005). The effects of an individualized imagery interventions on flow states and golf performance. Athletic Insight, 7(1), 43-66. Recuperado de https://eprints.qut.edu.au/106310/

Orwin, R. G. (1983). A fail-safe $\mathrm{N}$ for effect size in meta-analysis. Journal of Educational Statistics, 8(2), 157-159. Doi: https://doi.org/10.2307/1164923

Pain, M. A., Harwood, C., y Anderson, R. (2011). Pre-Competition Imagery and Music: The Impact on Flow and Performance in Competitive Soccer. The Sport Psychologist, 25(2), 212-

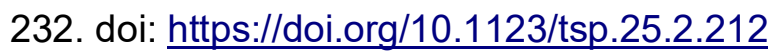


Pates, J, Karageorghis, C. I., Fryer, R., y Maynard, I. (2003). Effects of asynchronous music on flow states and shooting performance among netball players. Psychology of Sport and Exercise, 4(4), 415-427. doi: https://doi.org/10.1016/S1469-0292(02)00039-0

Pates, J., y Maynard, I. (2000). Effects of hypnosis on flow states and golf performance.

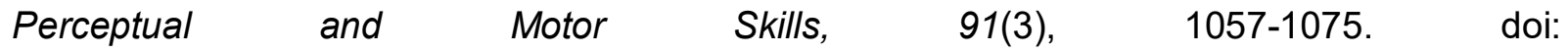
https://doi.org/10.2466/pms.2000.91.3f.1057

Pates, J. (2013). The effects of hypnosis on an elite senior European tour golfer: A single-subject design. The International Journal of Clinical and Experimental Hypnosis, 61(2), 193-204. doi: https://doi.org/10.1080/00207144.2013.753831

Pates, John, y Cowen, A. (2013). The Effect of a Hypnosis Intervention on Performance and Flow State of an Elite Golfer: A Single Subject Design. International Journal of Golf Science, 2(1), 43-53. Recuperado de https://www.golfsciencejournal.org/article/4957-the-effect-of-ahypnosis-intervention-on-performance-and-flow-state-of-an-elite-golfer-a-single-subjectdesign

Pates, J., Cowen, A. P., y Karageorghis, C. I. (2012). The Effect of a Client-Centered Approach on Flow States and the Performance of Three Elite Golfers. International Journal of Golf Science, 1(2), 113-126. doi: https://doi.org/10.1123/ijgs.1.2.113

Pates, J., Cummings, A., y Maynard, I. (2002). The effects of hypnosis on flow states and threepoint shooting performance in basketball players. The Sport Psychologist, 16(1), 34-47. doi: https://doi.org/10.1123/tsp.16.1.34

Pates, J., Oliver, R., y Maynard, I. (2001). The effects of hypnosis on flow states and golf-putting performance. Journal of Applied Sport Psychology, 13(4), 341-354. doi: https://doi.org/10.1080/104132001753226238 
Pates, J., y Palmi, J. (2002). The effects of hypnosis on flow states and performance. Journal of Excellence, (6), 48-62. Recuperado de https://hartpury.pure.elsevier.com/en/publications/theeffects-of-hypnosis-on-flow-states-and-performance

Sardon, D. Z. N. A., Mazaulan, M., y Mohamed, M. N. (2016). Effect of Imagery Intervention on Flow State and Performance in Tennis. En: S. Ismail, N. Sulaiman y R. Adnan (Eds.) Proceedings of the 2nd International Colloquium on Sports Science, Exercise, Engineering and Technology 2015 (ICoSSEET 2015) (pp. 269-280). Springer, Singapore. doi: https://doi.org/10.1007/978-981-287-691-1 29

Thomas, J. R., y French, K. E. (1986). The use of meta-analysis in exercise and sport: A tutorial. Research Quarterly for Exercise and Sport, 57(3), 196-204. doi: https://doi.org/10.1080/02701367.1986.10605397

Urrútia, G., y Bonfill, X. (2010). Declaración PRISMA: una propuesta para mejorar la publicación de revisiones sistemáticas y metaanálisis. Medicina clínica, 135(11), 507-511. doi: https://doi.org/10.1016/j.medcli.2010.01.015

Vasquez, B. L. (2005). The effects of hypnosis on flow and in the performance enhancement of basketball skills (Tesis doctorado). Washington State University. Recuperado de http://www.dissertations.wsu.edu/Dissertations/Fall2005/b vasquez 090805.pdf

Vealey, R. S., y Greenleaf, C. A. (2010). Seeing is believing: Understanding and using imagery in sport. En J. M. Williams (Ed.) Applied sport psychology: personal growth to peak performance (267-304). New York: Mc Graw-Hill Education. Recuperado de https://mdx.rl.talis.com/items/C5B8D9F0-CFE8-E452-A9DE-ED8F66792AFD.html

Welo, E. (2009). Mental Imagery and Basketball: A Comparison of Cognitive-specific and Flow Imagery. Recuperado de 
https://digitalcommons.macalester.edu/cgi/viewcontent.cgi?article=1016\&context=psychology honors 